\title{
Low-frequency broadband sound source localization using an adaptive normal mode back-propagation approach in a shallow-water ocean
}

\author{
Ying-Tsong Lin, ${ }^{\text {a) }}$ Arthur E. Newhall, and James F. Lynch \\ Applied Ocean Physics and Engineering Department, Woods Hole Oceanographic Institution, 266 Woods \\ Hole Road, Woods Hole, Massachusetts 02543
}

(Received 13 May 2011; revised 21 November 2011; accepted 27 November 2011)

\begin{abstract}
A variety of localization methods with normal mode theory have been established for localizing low frequency (below a few hundred $\mathrm{Hz}$ ), broadband signals in a shallow water environment. Gauss-Markov inverse theory is employed in this paper to derive an adaptive normal mode backpropagation approach. Joining with the maximum a posteriori mode filter, this approach is capable of separating signals from noisy data so that the back-propagation will not have significant influence from the noise. Numerical simulations are presented to demonstrate the robustness and accuracy of the approach, along with comparisons to other methods. Applications to real data collected at the edge of the continental shelf off New Jersey, USA are presented, and the effects of water column fluctuations caused by nonlinear internal waves and shelfbreak front variability are discussed. (C) 2012 Acoustical Society of America. [DOI: 10.1121/1.3672643]
\end{abstract}

PACS number(s): 43.60.Jn, 43.30.Bp, 43.60.Kx [AIT]

Pages: $1798-1813$

\section{INTRODUCTION}

Normal mode back-propagation is a physics-based signal processing technique which utilizes the propagation properties of low frequency sound (below a few hundred $\mathrm{Hz}$ ) in an ocean waveguide, and can be applied to target-localization. Acoustic normal mode propagation theory shows that low frequency sound propagation, especially in shallow water where the water depth is within the order of tens of meters, has significant acoustic mode dispersion (e.g., Frisk, 1994). Consequently, a received pulse on a hydrophone is comprised of several normal mode arrivals with separate arrival times, and the arrival time difference between each mode can provide useful information on the source-to-receiver distance. Additionally, the source depth can be estimated from the ratio of excitation of different modes or from the closure relation of normal modes. This paper presents an adaptive back-propagation approach employing Gauss-Markov inverse theory as well as adiabatic mode theory. This adaptive approach can adjust its kernel to balance robustness and accuracy depending on the uncertainty of the estimated mode arrivals.

Bucker (1976) first introduced Matched Field Processing (MFP) to utilize acoustic propagation physics via numerical models for source localization. Comprehensive discussions on a variety of MFP methods can be found in Baggeroer et al. (1993) and Tolstoy (1993). A back-propagation method can be considered as a variant of MFP due to the fact that it also makes use of phase conjugation. Instead of correlating the field with a model replica, phase conjugation in back-propagation is performed to reverse the waveguide dispersion. The application of backpropagation source localization was first introduced by Tappert et al. (1985). Their approach was to employ a parabolicequation (PE) propagation model to back-propagate the meas-

\footnotetext{
a) Author to whom correspondence should be addressed. Electronic mail: ytlin@whoi.edu
}

ured sound pressure on a vertical line array (Tappert et al., 1985). Voltz and Lu (1994) also established a back-propagation method using ray theory in the time domain. A more detailed overview of back-propagation techniques can be found in Meyer et al. (2006), and applications for geoacoustic inversion can be found in Dizaji et al. (2002) and Park et al. (2010).

Source localization using normal mode theory has drawn much attention in shallow water applications due to the modal nature of sound propagation. Shang (1985) and Shang et al. (1985) recognized that the source range and depth can be determined from the phase difference between modes and the closure relation of normal modes, respectively. Yang (1987) utilized jointly the phase conjugation and the closure relation for normal mode beamforming to source locations, which can also be considered as Matched Mode Processing (MMP). Yang (1990) showed that when the sampled-mode-shape mode filter (Ferris, 1972) is used, MMP is equivalent to MFP. One advantage of MMP is its tolerance to some environmental mismatch, since one can use a subset of modes that are less sensitive to the mismatch. However, when there are only a small number of modes observed or used, the closure relation holds only partially, causing some degradation of source depth resolution and often leading to biased estimates. To prevent this possible drawback, Shang (1989) proposed to use the ratio of mode amplitudes to achieve higher resolution. A potential disadvantage of this ratio method is its lack of signal-to-noise robustness. The ratio method requires a high signal-to-noise ratio, but the closure relation method does not. In this paper, an adaptive approach is presented to blend these two methods and procure the advantages of both.

Passive acoustic technology has been applied extensively to marine mammal monitoring (e.g. Clark et al., 2010), localization and tracking (e.g., Thode et al., 2000; Baumgartner et al., 2008). The normal mode back-propagation approach presented here has significant potential for improving longterm, long-range monitoring of baleen whales in coastal areas 
and on continental shelves, as it relies on only a single array sensor site. An example of this advantage is reported by Newhall et al. (2012) for tracking sei whales (Balaenoptera borealis) on the New Jersey shelf.

The paper is organized as follows. The method is explained in detail in Sec. II, and considerations for implementing the method are discussed in Sec. III. Simulation studies are shown in Sec. IV. To understand, calibrate and benchmark the technique in the real world, the method is employed in Sec. V to localize low-frequency broadband sources deployed during the Shallow Water 2006 (SW06) experiment (Tang et al., 2007) off the coast of New Jersey in 2006. Finally, conclusions and future directions are addressed in Sec. VI.

\section{NORMAL MODE BACK-PROPAGATION METHOD}

The normal mode back-propagation method utilizes modal dispersion to passively determine the source location with a basic assumption that the mode coupling is negligible so that adiabatic mode theory holds. The method is briefly described here. The first step is to implement a vertical mode filter to obtain individual modal arrivals from vertical hydrophone array data, and then back-propagate those modes with their own group speeds derived from the acoustic waveguide parameters. Back-propagation will reverse the modal dispersion, and the source position is estimated at the range where the back-propagated modes coincide with each other. For source depth estimation, two physical principles are utilized: (1) the ratio of excitation of different modes and (2) the normal mode closure relation. An adaptive method is proposed to allow a smooth transition from one to another depending on the signal-to-noise ratio. Source bearing is known prior to the back-propagation, as the bearing information can be obtained from beamforming a horizontal line array. In one of the examples shown in this paper, a horizontal beamforming technique employing normal mode theory will be shown.

The detailed methodology of this source localization method is provided in the rest of this section, and normal mode theory is reviewed first to provide some background.

\section{A. Normal mode theory}

Considering a broadband sound source at $\vec{x}_{s}$ with a source spectrum $S(\omega)$, the sound pressure time series $p(t)$ at a receiver at $\vec{x}_{r}$ can be expressed as an inverse Fourier transform

$$
p\left(\vec{x}_{r}, t\right)=\frac{1}{2 \pi} \int_{-\infty}^{\infty} S(\omega) G\left(\vec{x}_{r}, \omega ; \vec{x}_{s}\right) e^{-i \omega t} d \omega
$$

where $\omega$ is angular frequency, and $G\left(\vec{x}_{r}, \omega ; \vec{x}_{s}\right)$ is the Green's function between $\vec{x}_{s}$ and $\vec{x}_{r}$. From adiabatic mode/WKB theory (Frisk, 1994) one can get a two-dimensional (2-D) normal mode solution for the Green's function in a slowly varying environment:

$$
\begin{aligned}
G\left(\vec{x}_{r}, \omega ; \vec{x}_{s}\right)= & \sqrt{2 \pi} e^{i \pi / 4} \sum_{m} \frac{\Psi_{m}\left(z_{s} ; x_{s}, y_{s}, \omega\right)}{\rho\left(\vec{x}_{s}\right)} \Psi_{m}\left(z_{r} ; x_{r}, y_{r}, \omega\right) \\
& \times \frac{\exp \left\{\int_{\vec{x}_{s}}^{\vec{x}_{r}}\left[i \zeta_{m}(x, y, \omega)-\alpha_{m}(x, y, \omega)\right] d r\right\}}{\sqrt{\zeta_{m}\left(x_{r}, y_{r}, \omega\right) R}},
\end{aligned}
$$

where an auxiliary variable $r$ is used to denote the distance along the radial from the source to the receiver, and $0 \leq r \leq R$. $\Psi_{m}$ is the $m$ th mode function, $\zeta_{m}$ is its horizontal wavenumber, and $\alpha_{m}$ is its attenuation. The modes are obtained from the depth-dependent normal mode equation at each horizontal position with proper boundary conditions on the sea surface and the seafloor:

$$
\begin{aligned}
& \rho\left(z ; \vec{x}_{H}\right) \frac{d}{d z}\left[\frac{1}{\rho\left(z ; \vec{x}_{H}\right)} \frac{d}{d z} \Psi_{m}\left(z ; \vec{x}_{H}, \omega\right)\right] \\
& \quad+\left\{\frac{\omega^{2}}{c^{2}\left(z ; \vec{x}_{H}\right)}-\left[\zeta_{m}\left(\vec{x}_{H}, \omega\right)+i \alpha_{m}\left(\vec{x}_{H}, \omega\right)\right]^{2}\right\} \Psi_{m}\left(z ; \vec{x}_{H}, \omega\right)=0,
\end{aligned}
$$

where $\vec{x}_{H}$ indicates horizontal locations $(x, y)$, and $c$ is the complex sound speed (its imaginary part relates to medium absorption). The phase speed of a mode can be determined from $\omega / \zeta_{\mathrm{m}}$.

\section{B. Theoretical expressions}

There are different perspectives that we can take to look into the theory of this back-propagation method, but perhaps it is most useful to consider it as an estimation problem to determine the unknown source location from the modal arrivals. From Eqs. (1) and (2), the frequency spectrum of $p\left(\vec{x}_{r}, t\right)$ at $\omega$ is $P\left(\vec{x}_{r}, \omega\right)=\sum_{m} A_{m}\left(\vec{x}_{s}, \vec{x}_{r}, \omega\right) \Psi_{m}\left(\vec{x}_{r}, \omega\right)$, and the amplitude spectrum of mode $m, A_{m}$, can be readily found as

$$
\begin{aligned}
& A_{m}\left(\vec{x}_{s}, \vec{x}_{r}, \omega\right)=A_{0} S(\omega) E_{m}\left(\vec{x}_{s}, \vec{x}_{r}, \omega\right), \\
& \text { where }\left\{\begin{aligned}
A_{0}= & \sqrt{2 \pi} e^{i \pi / 4} \cdot \rho^{-1}\left(\vec{x}_{s}\right) \cdot R^{-1 / 2} \\
E_{m}= & \Psi_{m}\left(\vec{x}_{s}, \omega\right) \cdot \zeta_{m}^{-1 / 2}\left(x_{r}, y_{r}, \omega\right) \\
& \times \exp \left[\int_{\vec{x}_{s}}^{\vec{x}_{r}} i \zeta_{m}(x, y, \omega)-\alpha_{m}(x, y, \omega) d r\right] .
\end{aligned}\right.
\end{aligned}
$$

$E_{m}$ is essentially a mode content function and contains the mode functions at the source location $\Psi_{m}\left(\vec{x}_{s}, \omega\right)$ reflecting the modal excitation, and thus has source depth information. Additionally, it has an exponential function describing the modal dispersion, which contains source range information. By back-propagating each modal arrival and reversing its modal excitation and dispersion, we should, in theory, obtain the same scaled source function $A_{0} S(\omega)$ from each backpropagated mode at the true source location.

Reversing modal excitation and dispersion at each backpropagation step $\vec{x}_{b}$ can be done mathematically by inverting the mode content function. The most apparent approach is to take a direct inversion of $E_{m}\left(\vec{x}_{b}, \vec{x}_{r}, \omega\right)$, which results in a spectrum of the back-propagated mode: $B_{m}\left(\vec{x}_{b}, \omega\right)$ $=E_{m}^{-1}\left(\vec{x}_{b}, \vec{x}_{r}, \omega\right) A_{m}(\omega)$. Note that the variable of source position in $E_{m}$ is now $\vec{x}_{b}$. Assuming the modes are not coupled, the normal mode back-propagation can be done for each mode independently. Again, when $\vec{x}_{b}=\vec{x}_{s}$ we recover the same source spectrum, scaled by $A_{0}$, from all of the modes. 
One immediate shortcoming of this direct inversion is that it becomes singular when $E_{m}\left(\vec{x}_{b}, \vec{x}_{r}, \omega\right)$ is zero. To avoid the singularity, one can use a pseudo-inverse in linear algebra, where $0 / 0$ is defined to be 0 . In doing so, we first discretize the frequency into $\Omega$ bins, which naturally results from employing a Discrete Fourier Transform or a Fast Fourier Transform (FFT) on a time series. We then rewrite Eq. (4) into a matrix form of $\mathbf{A}_{m}=A_{0} \mathrm{E}_{m} \mathbf{S}$, where $A_{0}$ is still a scalar, and the size of the other variable vectors/matrices is $\Omega \times 1$ for both $\mathbf{S}$ and $\mathbf{A}_{m}$, and $\Omega \times \Omega$ for $\mathbf{E}_{m}$ which will be a diagonal matrix when the Doppler shift is not considered. The pseudoinverse back-propagation can then be formulated as

$$
\mathbf{B}_{m}\left(\vec{x}_{b}\right)=\left\{\mathbf{E}_{m}^{\mathrm{H}}\left(\vec{x}_{b}\right) \mathbf{E}_{m}\left(\vec{x}_{b}\right)\right\}^{-1} \mathbf{E}_{m}^{\mathrm{H}}\left(\vec{x}_{b}\right) \mathbf{A}_{m},
$$

where the notation for frequency is dropped for conciseness. This pseudo-inverse does overcome the singularity problem. However, it is still very sensitive to the noise or uncertainties in the modal amplitude when the condition number of $\mathbf{E}_{m}$ is large, which will happen for example, when the signal is backpropagated to a depth close to a node (zero) of a local mode. To obtain a more robust inversion, one can use a weighted and tapered least squares inversion (Wunsch, 1996), which gives

$$
\mathbf{B}_{m}\left(\vec{x}_{b}\right)=\left\{\mathbf{E}_{m}^{\mathrm{H}}\left(\vec{x}_{b}\right) \mathbf{W}_{m}^{-1} \mathbf{E}_{m}\left(\vec{x}_{b}\right)+\mathbf{U}_{m}^{-1}\right\}^{-1} \mathbf{E}_{m}^{\mathrm{H}}\left(\vec{x}_{b}\right) \mathbf{W}_{m}^{-1} \mathbf{A}_{m},
$$

where $\mathbf{U}_{m}$ and $\mathbf{W}_{m}$ are column and row scaling matrices, respectively, and the superscript $\mathrm{H}$ indicates the Hermitian transpose. Choosing the scaling matrices is somewhat arbitrary in the sense of least squares, but for getting maximum a posteriori estimates it is natural to make the ratio of the scaling matrices $\left\|\mathbf{U}_{m}\right\| /\left\|\mathbf{W}_{m}\right\|$ proportional to the signal-tonoise ratio of the modal amplitude estimates. We will discuss the selection of scaling matrices in detail later.

Normal mode back-propagation with Eq. (6) is adaptively adjusted by the signal-to-noise ratio (SNR). Although we have not yet detailed the scaling matrices involved in the equation, their ratio $\left\|\mathbf{U}_{m}\right\| /\left\|\mathbf{W}_{m}\right\|$ should be proportional to the SNR of the modal amplitude estimates. In this case, if the SNR is low, Eq. (6) will approach $\mathbf{E}_{m}^{\mathrm{H}}\left(\vec{x}_{b}\right) \mathbf{A}_{m}$, and it still has the capability to resolve the source location. This mode conjugate formula is used in Matched Mode Processing (Yang, 1987; Yang, 1990). The principal criterion for ranging the source is phase conjugation, and the closure relation of the normal modes, i.e., $\Sigma_{m} \Psi_{m}^{\mathrm{H}}(z) \Psi_{m}\left(z_{s}\right)=\delta\left(z-z_{s}\right)$, is employed for estimating the source depth. Note that the closure relation holds completely only when there is an infinite set of modes (propagating plus continuum and evanescent modes). In shallow water, where we have a finite set of propagating modes in the far field, this closure relation holds only partially, and so it loses some resolution for determining the source depth. However, it is still a robust depth estimator. On the other hand, when the SNR is high, Eq. (6) approaches to a pseudo-inverse, and the ratio of modal excitations becomes the key for source depth estimation. This will be shown later in a simulation example to have superior resolution, even when just considering only a few propagating modes.
The inversion kernel varies with back-propagation steps because of its dependence on $\vec{x}_{b}$. Thus, there is a series of inversions being implemented in the back-propagation procedure. To determine the source location from these inversions, we can use the difference between each backpropagated mode as a metric (minimal difference gives us the source location), since we are expecting identical backpropagated modes at the source location. Alternatively, we can also examine their cross-correlations and select the maximal correlation point as the source location. The next algebraic equality shows that these two criteria can actually produce the same cost function for source localization.

$$
\frac{\sum_{m \neq n} \mathbf{B}_{m}^{\mathrm{H}} \mathbf{B}_{n}}{\sum_{m}\left\|\mathbf{B}_{m}\right\|^{2}}+1=\mathbf{M}-\frac{1}{2} \frac{\sum_{m \neq n}\left\|\mathbf{B}_{m}-\mathbf{B}_{n}\right\|^{2}}{\sum_{m}\left\|\mathbf{B}_{m}\right\|^{2}}=\frac{\left\|\sum_{m} \mathbf{B}_{m}\right\|^{2}}{\sum_{m}\left\|\mathbf{B}_{m}\right\|^{2}},
$$

where $\mathbf{B}_{m}^{H} \mathbf{B}_{n}$ is the cross-correlation with zero-lag between back-propagated modes $m$ and $n$, and $\left\|\mathbf{B}_{m}-\mathbf{B}_{n}\right\|^{2}$ is the squared $l^{2}$ norm of their difference. $M$ is the number of backpropagated modes, and $\sum_{m}\left\|\mathbf{B}_{m}\right\|^{2}$ is their total intensity, acting like a normalization factor. The first equality in Eq. (7) actually indicates that maximizing the cross-correlation is the same as minimizing the difference. The second equality in Eq. (7) suggests that we can select the coherent sum of back-propagated modes to be the cost function, i.e.,

$$
C_{\gamma}\left(\vec{x}_{b}\right)=\frac{\left\|\sum_{m} \mathbf{B}_{m}\left(\vec{x}_{b}\right)\right\|^{2}}{\sum_{m}\left\|\mathbf{B}_{m}\left(\vec{x}_{b}\right)\right\|^{2}}
$$

and the final source location estimate is $\hat{x}_{s}$ $=\arg \max _{\vec{x}_{b} \in \vec{x}} \mathcal{C}_{\gamma}\left(\vec{x}_{b}\right)$. Note that computing the coherent sum requires less algebraic manipulations than calculating the cross-correlation or the inter-mode difference. It also provides an intuitive index for the similarity of backpropagating modes. For example, when the cost function reaches its maximum $M$, it indicates that all of $M$ backpropagated modes are identical.

\section{IMPLEMENTATION CONSIDERATIONS}

In this section, how to use a vertical mode filter to estimate modal amplitudes will be explained. In practice, acoustic noise and array tilt will produce errors in the modal amplitude estimates, and we will discuss how to reduce these errors. The noise issue is tied with the scaling matrices, $\mathbf{U}_{m}$ and $\mathbf{W}_{m}$, used in the back-propagation inversion. The impact of mode coupling and three-dimensional (3-D) propagation effects will also be discussed.

\section{A. Modal amplitude estimation: Normal mode filtering with a vertical line array}

The first step in the implementation of the backpropagation method is to perform a mode filter to obtain 
individual modal arrivals (modal amplitudes), which can be done using a vertical hydrophone array. Since broadband signals and the frequency dependence of normal modes are considered, it is easier to perform mode filtering in the frequency domain. This technique is well established and is reviewed below with some discussion on its application to normal mode back-propagation.

As a vertical hydrophone array receives broadband sound at different depths, the acoustic data space spans both time/ frequency and spatial/wavenumber domains. Thus, the sampled sound field can be decomposed by spatial basis functions (normal modes) at each frequency, i.e., $P\left(z_{r}, \omega\right)$ $=\sum_{m} A_{m}(\omega) \Psi_{m}\left(z_{r}, \omega\right)$. The goal of mode filtering is to obtain the modal amplitudes $A_{m}(\omega)$, and this can be done with linear inverse theory. We can rewrite the modal decomposition at each frequency in matrix form $\mathbf{P}_{\mathrm{N} \times 1}\left(\omega_{i}\right)$ $=\Psi_{\mathrm{N} \times \mathrm{M}}\left(\omega_{i}\right) \mathrm{A}_{\mathrm{M} \times 1}\left(\omega_{i}\right)$, where the number of frequencies $\omega_{i}$ is $\Omega$, and $\mathrm{N}$ hydrophones on the array and $\mathrm{M}$ modes are assumed in the decomposition. There should be $\Omega$ such equations within the frequency band, and if we consider the spatial-spectral cross-correlation of the acoustic signal and noise, we need to form a full matrix equation by cascading the variable vectors and matrices. Many inversion methods can be used in solving this linear mode filtering equation, such as direct projection with sampled mode shapes (Ferris, 1972), pseudo-inverse (Tindle et al., 1978) and Gauss-Markov estimation (Chiu et al., 1997; Buck et al., 1998). The direct projection method is optimal for detecting a single mode in a white noise field, but it suffers from modal cross-talk in resolving multiple modes. The pseudo-inverse method can preserve the orthogonality of normal modes, hence it produces the least modal cross-talk. However, it is sensitive to noise, as is observed in the pseudo-inverse back-propagation Eq. (5). The Gauss-Markov estimator leads to a maximum a posteriori mode filter (Chiu et al., 1997; Buck et al., 1998) when modal amplitudes and noise are zero-mean complex Gaussian random variables:

$$
\hat{\mathbf{A}}_{\mathrm{M} \times 1}=\left\{\boldsymbol{\Psi}_{\mathrm{N} \times \mathrm{M}}^{\mathrm{H}} \mathbf{R}_{n n}^{-1} \boldsymbol{\Psi}_{\mathrm{N} \times \mathbf{M}}+\mathbf{R}_{A A}^{-1}\right\}^{-1} \boldsymbol{\Psi}_{\mathrm{N} \times \mathrm{M}}^{\mathrm{H}} \mathbf{R}_{n n}^{-1} \mathbf{P}_{\mathrm{N} \times 1},
$$

where $\mathbf{R}_{A A}$ and $\mathbf{R}_{n n}$ are the expected second moments, $a$ priori information about the modal amplitudes and noise, respectively. From the theory of Gauss-Markov estimation, the posteriori uncertainty of amplitude estimates can be found to be $\left\{\boldsymbol{\Psi}_{\mathrm{N} \times \mathrm{M}}^{\mathrm{H}} \mathbf{R}_{n n}^{-1} \boldsymbol{\Psi}_{\mathrm{N} \times \mathrm{M}}+\mathbf{R}_{\mathrm{AA}}^{-1}\right\}^{-1}$. Note that this estimation is made under an assumption of statistical stationarity, which means $\mathrm{R}_{n n}$ and $R_{A A}$ are considered to be constant in the random process, or at least over a certain period. Chiu et al. (1997) and Buck et al. (1998) showed that this mode filtering technique can reach the optimal balance between rejecting the noise and preserving the orthogonality of normal modes. This is due to the fact that this estimation takes into account the statistics both of noise and signals, and so enables us to separate noise and signals. A complete Gauss-Markov estimation should also involve a posteriori test to check if the behavior of the estimated noise, i.e., residues of the filter, is consistent with the prior statistics.
Estimation of $\mathbf{R}_{n n}$ and $\mathbf{R}_{A A}$ is necessary for implementation of a Gauss Markov mode filter, but it often suffers from snapshot deficiency, which causes the sampled $\mathbf{R}_{n n}$ and $\mathbf{R}_{A A}$ matrices to be not full-rank. Many methods (e.g., Song et al., 2003 , and references therein) have been proposed to overcome this problem, and a method of replacing zero eigenvalues with the minimal non-zero eigenvalue has been found very useful in the application of broadband normal mode filtering. Instead of truncating the eigenvector null space, preserving them with the least weight (the minimal non-zero eigenvalue) ensures that the part of the signal or noise in the null space is still being properly treated in the mode filtering inversion. A more detailed discussion on this will be given when examples are presented later.

Array tilt also needs to be corrected for mode filtering, or otherwise unwanted modal cross-talk will occur. Given horizontal deviations of array elements $\Delta x_{n}$, a phase correction of $\zeta_{m} \Delta x_{n}$ can be applied in the mode function matrix $\boldsymbol{\Psi}_{\mathrm{N} \times \mathrm{M}}$ in Eq. (9), i.e.,

$$
\Psi_{n m}^{\prime}=\Psi_{n m} \exp \left(-i \zeta_{m} \Delta x_{n}\right)
$$

If the exact array tilt is not available, a total least squares inversion (Markovsky and Van Huffel, 2007) can be used to account for the array tilt uncertainty in the mode function matrix. The details of this inverse method are beyond the scope of this paper, and we shall assume in the following examples that some reliable array tilt estimates are available. In fact, from a modeling study shown later, the source localization method with normal mode back-propagation is tolerant of array tilt to a certain degree so that one may sometimes disregard the tilt correction in comparison to other environmental uncertainties in the ocean.

\section{B. Selection of the scaling matrices for the back-propagation inversion}

To avoid an ill-conditioned inversion for back-propagating modes, one should take the approach of weighted and tapered least squares. The following is a discussion on selecting the scaling matrices for the back-propagation inversion.

Recall Eq. (6), $\quad \mathbf{B}_{m}\left(\vec{x}_{b}\right)=\left\{\mathbf{E}_{m}^{\mathrm{H}}\left(\vec{x}_{b}\right) \mathbf{W}_{m}^{-1} \mathbf{E}_{m}\left(\vec{x}_{b}\right)\right.$ $\left.+\mathbf{U}_{m}^{-1}\right\}^{-1} \mathbf{E}_{m}^{\mathrm{H}}\left(\vec{x}_{b}\right) \mathbf{W}_{m}^{-1} \mathbf{A}_{m}$. When the second moment of $A_{0} \mathbf{S}$ is used for $\mathbf{U}_{m}$, and the modal amplitude uncertainty is used for $\mathbf{W}_{m}$, this inversion is exactly a Gauss-Markov estimation. In fact, if the Gauss-Markov mode filter mentioned above is also used for estimating modal amplitudes $\mathbf{A}_{m}$, the posterior uncertainty of modal amplitude estimates is readily available [see Eq. (9) and its discussion] and can be inherently used for the row-scaling matrix $\mathbf{W}_{m}$. On the other hand, it requires some effort to obtain the column-scaling matrix $\mathbf{U}_{m}$. Due to the fact that the back-propagation process mentioned above only reverses the modal excitation and dispersion without restoring the spreading loss, the second moment of $A_{0} \mathbf{S}$ is suggested for $\mathbf{U}_{m}$. However, when the source spectrum $\mathbf{S}$ is unknown, it is not clear how the second moment of $A_{0} S$ can be determined. A useful approach is provided below.

In deriving the scaling matrix $\mathbf{U}_{m}$ directly from the modal amplitude estimates without prior knowledge about 
the source function, we first consider the relationship between the magnitude of the modal amplitude and the source function, i.e., $\left|A_{m}\left(\vec{x}_{r}\right)\right|=\left|A_{0} S\right| \cdot\left|\Psi_{m}\left(\vec{x}_{s}\right)\right| \cdot \zeta_{m}^{-1 / 2}\left(\vec{x}_{r}\right)$ with an assumption of small modal attenuation. If the mode function at the unknown source position has a similar structure to the one at the receiver, we can use the following scaling matrix for the case where the probability distribution of source depth is uniform over the entire water column:

$$
\mathbf{U}_{m, \Omega \times \Omega}=\operatorname{diag}\left(\frac{\zeta_{m}(\omega)\left|\hat{A}_{m}(\omega)\right|^{2}}{D^{-1} \int_{0}^{D}\left|\Psi_{m}(z)\right|^{2} d z}\right),
$$

where $D$ is the water depth at receiver, and the integral in the denominator is due to the uniform distribution of unknown source depth. One can see that the formulated $\mathbf{U}_{m}$ does not have off-diagonal terms, which indicates that the cross spectrum is not considered for back-propagation, but it also does not require prior knowledge about the source function. Although the cross spectrum is not considered here, the Gauss-Markov mode filter does account for that. Denoting the diagonal element of $\mathbf{U}_{m}$ as $s^{2}(\omega)$, and neglecting the cross spectral components of modal amplitude uncertainty, the back-propagation inversion Eq. (6) can be further simplified:

$$
\begin{aligned}
B_{m}\left(\vec{x}_{b}, \omega\right)= & \frac{Z_{m}^{*}\left(\vec{x}_{b}, \omega\right)}{\left|Z_{m}\left(\vec{x}_{b}, \omega\right)\right|^{2}+\frac{\sigma^{2}(\omega)}{s^{2}(\omega)}} \exp \left[-i \int_{\vec{x}_{b}}^{\vec{x}_{r}} \zeta_{m}(\vec{x}, \omega) d r\right] \\
& \times \hat{A}_{m}\left(\vec{x}_{b}, \omega\right)
\end{aligned}
$$

where, $Z_{m}\left(\vec{x}_{b}, \omega\right)=\Psi_{m}\left(\vec{x}_{b}, \omega\right) \zeta_{m}^{-1 / 2}\left(\vec{x}_{r}, \omega\right) \exp \left[-\int_{\vec{x}_{b}}^{\vec{x}_{r}} \alpha_{m}(\vec{x}, \omega) d r\right]$, and $\sigma^{2}(\omega)$ are the diagonal elements of the modal amplitude uncertainty. The loading term $\sigma^{2} / s^{2}$ is in fact the ratio $\left\|\mathbf{W}_{m}\right\| /$ $\left\|\mathbf{U}_{m}\right\|$, and this back-propagation inversion will have the adaptive capability mentioned in the discussion of Eq. (6).

\section{Mode coupling and 3-D propagation effects}

The scattering and refraction of sound from the ocean and seabed can create two error effects for our adiabatic back-propagation method, mainly time wander and time spreading of the arriving signal. The time wander of the signal is probably negligible, since the time wander seen in shallow water applications (e.g., Headrick et al., 2000a,b) is on the order of $10 \mathrm{msec}$, which would translate into a small ranging error $(\sim 15 \mathrm{~m})$. Also, wander is essentially not a mode coupling effect, so it poses little problem against our adiabatic mode assumption. Error caused by the time spread of the signal due to either mode coupling or 3-D propagation effects is more serious, in that it can "smear" the data over the effective length of the entire sequence of modal arrivals and make the individual modal arrivals unresolvable. For our technique, which relies on adiabatic mode theory to allow modal "realignment," this will be entirely debilitating. Once the modal arrivals become unresolvable in time, the data become useless for this technique.

Time spreading will affect the performance of the backpropagation method, and the exact amount of time spreading will vary widely, depending upon the range, frequency, and physical environment through which the sound propagates and scatters. Since the exact calculations of such broadband, coupled mode or 3-D propagation are rather tedious, we will discuss the spreading effects here rather than perform detailed calculations.

3-D oceanography and topography produce a spreading of the signal due to the introduction of additional horizontal multipaths, which can be mode or ray in nature. For each vertical acoustic normal mode that we calculate for a twodimensional path between source and receiver, there can be a number of horizontal modes as well, so that a vertical mode indexed by $m$ now becomes indexed by $m n$, where the $n$ indexes the added horizontal modes. These $m n$ modes arrive at different times (i.e., they have slightly different group speeds), so that instead of a single mode $m$, multiple arrivals will appear and spread out over time. Modal arrivals are often tightly spaced in time, so any additional arrivals may overlap and make identification and resolution of modes in time impossible. Angular resolution can help here in theory, though the angles between the 3-D multipaths are often quite small.

Time spreading caused by mode coupling is easy to assess as to the maximum spread, which is (ignoring the continuum) the time between the fastest and slowest trapped mode's arrival. Much harder to assess is the strength of the mode coupling, as this involves the evaluation of a fairly detailed overlap integral involving the initial and final modal states and the environmental perturbation creating the coupling. We will not evaluate such integrals here, as it is beyond the scope of this paper, and the readers are referred to Headrick et al. (2000a,b) for detailed discussion. A numerical example will be presented later in the paper to demonstrate the impact of mode coupling caused by nonlinear internal waves on the back-propagation method.

\section{SIMULATION STUDIES}

Numerical examples of source localization using the normal mode back-propagation are presented in this section. The sound speed profile used here is shown in Fig. 1(a), along with the source function model, which consists of eight cycles of $100 \mathrm{~Hz}$ sinusoidal waves tapered with a Hann window. From the source spectrum shown in Fig. 1(a), one can see that the first pair of nulls around the center frequency are at $75 \mathrm{~Hz}$ and $125 \mathrm{~Hz}$, and the frequency band considered in the example is taken as between these two nulls. The sound speed profile is in fact the mean profile derived from data collected during the SW06 experiment (Lin et al., 2010), presenting a general sound speed profile for summer at the edge of the continental shelf off New Jersey, USA. The water depth is $80 \mathrm{~m}$, and the modeled bottom is homogeneous, with sound speed $1700 \mathrm{~m} / \mathrm{s}$, density $1.5 \mathrm{~g} / \mathrm{cm}^{3}$ and attenuation $0.5 \mathrm{~dB}$ per wavelength.

Figure 1(b) presents the signals in the numerical model. The hydrophone array in the model is located $20 \mathrm{~km}$ from the source, and it has 13 hydrophones spanning the water column from $10 \mathrm{~m}$ to $70 \mathrm{~m}$ with equal spacing. Modal arrivals at the array and their back-propagated modes are also 

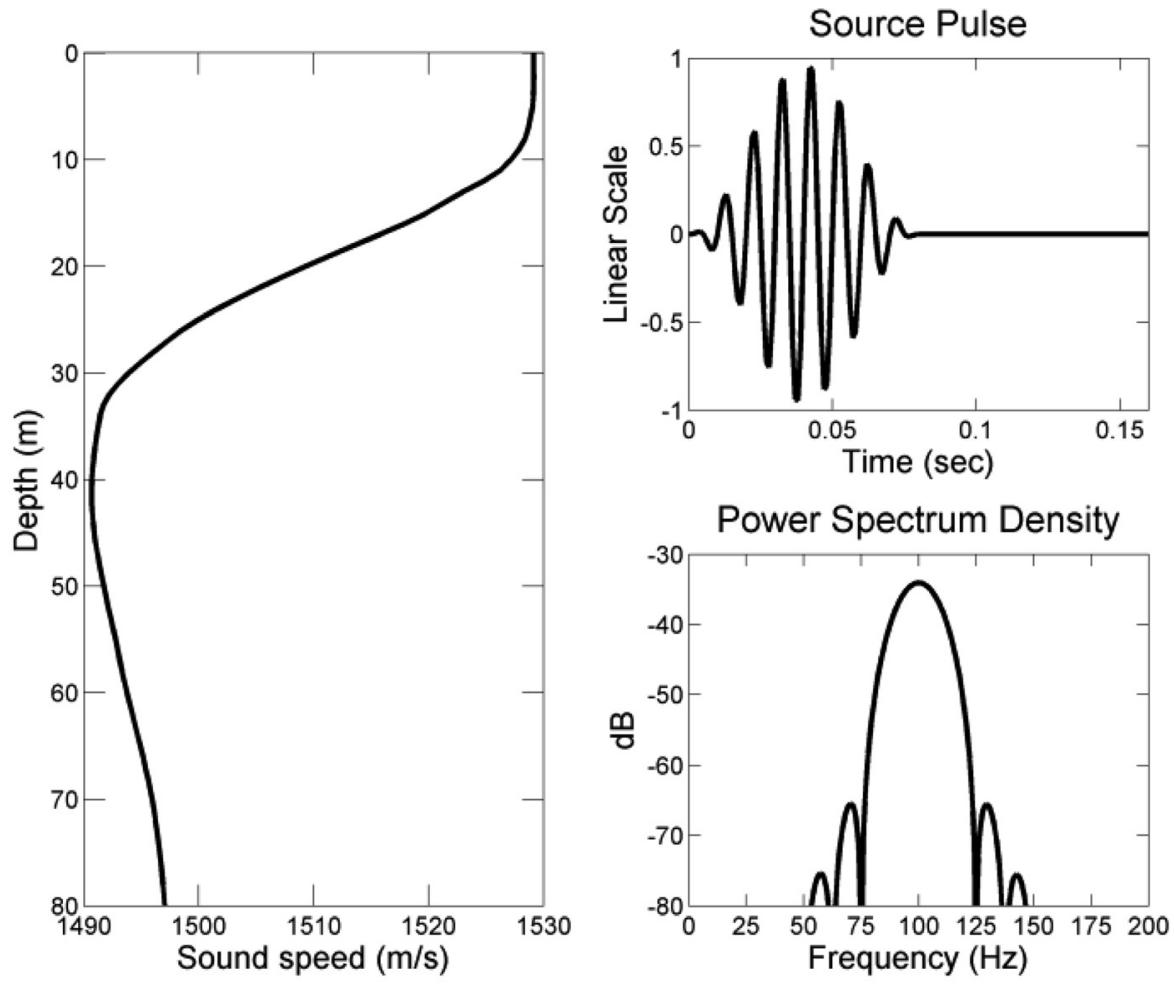

(a)

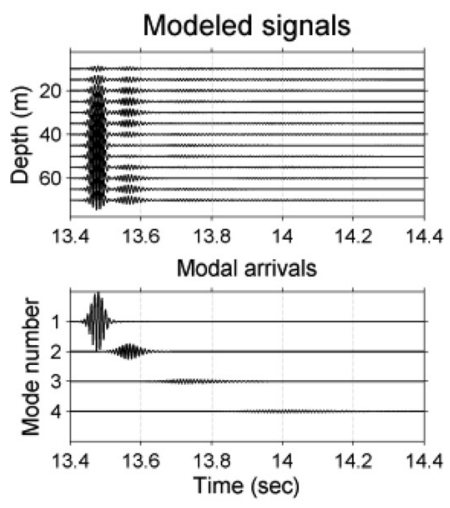

Back-propagate for $15 \mathrm{~km}$

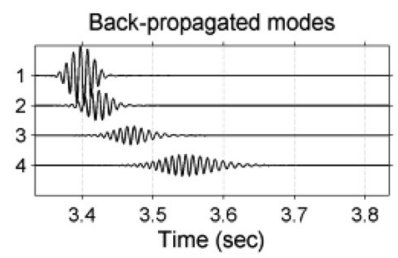

(b)
Back-propagate for $18 \mathrm{~km}$

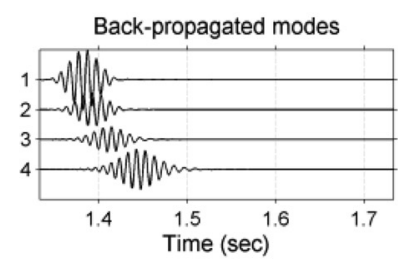

FIG. 1. (a) Sound speed profile and source function used in the numerical examples. (b) Modeled signals on the hydrophone array located $20 \mathrm{~km}$ away from the source are shown in the upper left panel, and the modal arrivals at the receiver are shown in the left panel. The right panels present the modes when they are back-propagated for $15 \mathrm{~km}$ and $18 \mathrm{~km}$. shown in Fig. 1(b). The modes at the array are dispersed, but when they are back-propagated, they become closer to each other, indicating that the modal dispersion has been compensated for. The modeled signal is considered to be deterministic, which means there is no random variability in the modeled environment and source function. This is to simplify the method comparisons presented below. In fact, the back-propagation approach can be applied to stochastic signals, as will be seen in the SW06 examples in Sec. V.

\section{A. Modeling methods}

Modeling examples are shown with the aim of testing the back-propagation method for four factors: (1) localization resolution, (2) noise tolerance, (3) effects of array tilt, and (4) effects of nonlinear internal waves. Two other established methods of localization are also compared here. The first method is Bartlett matched-field processing (MFP) and the second one is Minimum Variance Distortionless Response (MVDR) matched-field processing (Baggeroer et al., 1993). These two MFP's can be in general formulated as

$$
C_{\mathrm{MFP}}\left(\vec{x}_{b}\right)=\left|\mathbf{w}^{\mathrm{H}}\left(\vec{x}_{b}\right) \mathbf{d}\right|^{2}=\mathbf{w}^{\mathrm{H}}\left(\vec{x}_{b}\right) \mathbf{R}_{d d} \mathbf{w}\left(\vec{x}_{b}\right)
$$

where $\mathbf{d}$ is a data vector consisting of the received signal $\mathbf{p}$ and noise $\mathbf{n}$, thus $\mathbf{R}_{d d}=\boldsymbol{R}_{p p}+\mathbf{R}_{n n}$, and the data vector is formed by cascading the data (frequency spectra) received by each hydrophone on an array. The processing output $C_{\mathrm{MFP}}$ can be considered as the output power, and the steering vector $\mathbf{w}$ is formed from the theoretical prediction $\hat{\mathbf{p}}$ of the received signal, the socalled replica, assuming the source location is at $\vec{x}_{b}$. The steering vector of the Bartlett MFP is simply the replica without considering the noise, i.e., $\mathbf{w}_{\text {Bart }}\left(\vec{x}_{b}\right)=\hat{\mathbf{p}}\left(\vec{x}_{b}\right)$. On the other hand, the steering vector of the MVDR MFP does consider the noise, and it is $\mathbf{w}_{\mathrm{MVDR}}\left(\vec{x}_{b}\right)=\mathbf{R}_{d d}^{-1} \hat{\mathbf{p}}\left(\vec{x}_{b}\right)\left[\hat{\mathbf{p}}^{H}\left(\vec{x}_{b}\right) \mathbf{R}_{d d}^{-1} \hat{\mathbf{p}}\left(\vec{x}_{b}\right)\right]^{-1}$. The 
Bartlett MFP tolerates noise adequately, but its resolution is not very good for estimating source depth. The MVDR method can produce superb resolution when the data covariance $\mathbf{R}_{d d}$ is perfectly known, but this is extremely difficult to achieve in a real case.

Implementing the MVDR MFP and the backpropagation method with the Gauss-Markov mode filter requires the inverse of the covariance matrices. In the case of snapshot deficiency, the sampled covariance matrix is not full rank, and its inverse matrix will not exist. In the following examples, the covariance $\mathbf{R}_{d d}$ is given exactly for the MVDR MFP, and makes it ideal and perfect. On the other hand, the exact information about the signal and noise is intentionally not provided to the back-propagation, and so will make its comparison to the ideal MVDR MFP more critical.

Fifty realizations of the modeled signal plus Gaussian noise with a given SNR are made for localization in each test, and the same set of modeled data (signal plus noise) is used in all methods for consistent comparisons. The noise intensity is kept constant at each water depth, and the SNR value is determined by comparing the constant noise to the depth-averaged signal intensity in the water column. When implementing the back-propagation approach, the pseudo-inverse mode filter is employed first on these 50 realizations of noisy data, and the resultant mode estimates are coherently averaged to provide an estimate $\hat{\mathbf{R}}_{A A}$ for the second moment of the modal amplitude. In addition, 50 realizations of Gaussian random noise are generated separately, and the method of replacing zero eigenvalues with the minimal non-zero eigenvalue, described in Sec. III A, is employed to regularize the sampled noise covariance matrix $\hat{\mathbf{R}}_{n n}$. Since the signal in the numerical examples is assumed to be deterministic, the true $\mathbf{R}_{A A}$ is a rank-one matrix equal to $\mathbf{A} \mathbf{A}^{\mathrm{H}}$, and it is not invertible. Thus, another form of the GaussMarkov estimator for mode filtering is used replacing Eq. (9):

$$
\hat{\mathbf{A}}=\mathbf{R}_{A A} \boldsymbol{\Psi}^{\mathrm{H}}\left\{\boldsymbol{\Psi} \mathbf{R}_{A A} \boldsymbol{\Psi}^{\mathrm{H}}+\mathbf{R}_{n n}\right\}^{-1} \mathbf{P} .
$$

The exact source function is given for the Bartlett and MVDR MFP's in the examples, but not for the backpropagation approach. The scaling matrices described in Sec. III B are employed for back-propagation inversion, and they do not require knowledge about the source function. When implementing the Bartlett MFP, the modeled data (signal plus noise) is normalized in such a way that its total power equals to one i.e., $\mathbf{d}^{\mathrm{H}} \mathbf{d}=1$. The steering vector is also normalized in the same way, $\mathbf{w}_{\text {MFP }}^{\mathrm{H}} \mathbf{W}_{\mathbf{M F P}}=1$. This makes the maximal output of the Bartlett MFP equal to one. In the following back-propagation examples, four modes are being used and back-propagated to localize the source.

\section{B. Localization resolution}

The first test is focused on the resolution of localization. A high $20 \mathrm{~dB}$ SNR is considered here, as the noise tolerance is tested separately later. With a relatively high SNR, the MVDR MFP can produce superb resolution; hence it is not used for the explicit comparisons presented here.

A source is placed at a distance $20 \mathrm{~km}$ away from the receiving array. Three different source depths (15, 35, and
$55 \mathrm{~m}$ ) are considered, and the back-propagation approach with four modes and the Bartlett MFP are implemented. Figure 2 shows the ambiguity plots of source location estimate, and both methods produce a maximal peak at the true source location. Recall that the maximal peak height in the backpropagation approach is the number of back-propagated modes and is one in the Bartlett MFP with the normalization mentioned previously. Comparing the ambiguity plots shows that the back-propagation generally has much better depth resolution than the Bartlett MFP. Note that the peak shapes of both methods in the case of $15 \mathrm{~m}$ source depth are comparable, but the back-propagation peak is still slightly sharper. Given that this shallow depth is near the edge of the mixed layer [see Fig. 1(a)] and the source range is $20 \mathrm{~km}$, this slight improvement is appreciable. Comparing the sharpness of the peaks for range resolution reveals that the Bartlett MFP is just slightly better, but it has significant side lobes at distance $\sim 18.5$ and $21.5 \mathrm{~km}$.

Figure 3 presents the detailed comparisons of depth resolution in the case of $55 \mathrm{~m}$ source depth. Figure 3(a) shows that the main lobe of the back-propagation kernel is much sharper than the Bartlett MFP. Additionally, the side lobes of the back-propagation kernel are in general smaller than the envelope of the Bartlett MFP kernel. Figure 3(b) shows a comparison of the resolution produced by the backpropagation approach with a different number of modes. The resolution resulting from using three modes is nearly as good as using four modes.

\section{Noise tolerance}

This test focuses on the noise tolerance of the backpropagation approach and also presents the comparisons to the Bartlett and MVDR MFP localization. Four different SNR's, $20 \mathrm{~dB}, 10 \mathrm{~dB}, 0 \mathrm{~dB}$, and $-10 \mathrm{~dB}$ are considered here, along with source locations at depths of $15 \mathrm{~m}, 35 \mathrm{~m}$, and $55 \mathrm{~m}$. The same source range is considered at $20 \mathrm{~km}$. In each test with a given pair of SNR and source depth, 50 realizations of signal plus noise are generated for the localization.

Table I summarizes the numerical results of this noise tolerance example. At $20 \mathrm{~dB}$ SNR, all of the methods successfully locate the source with zero standard deviation. When the SNR slightly decreases to $10 \mathrm{~dB}$, the MVDR MFP starts suffering from the noise and loses accuracy and robustness. However, the back-propagation approach and Bartlett MFP still perform well. A slight bias in the source depth estimate is observed in the results of back-propagation localization. At $0 \mathrm{~dB}$, the MVDR MFP suffers greatly, and its estimates of source depth are no longer correct, and significant variance is seen in the source range estimates. Comparing the results from back-propagation and Bartlett MFP, one can also see that the back-propagation approach has smaller variance in estimating source depth with only a slightly degraded accuracy. The source range estimates from these two methods are still accurate and robust. When the SNR goes down to $-10 \mathrm{~dB}$, the performance of the MVDR MFP is as bad as the $0 \mathrm{~dB}$ case, indicating the influence of noise on this method is saturated. The results indicate the Bartlett MFP reaches the limit of its noise tolerance. As for the back-propagation 

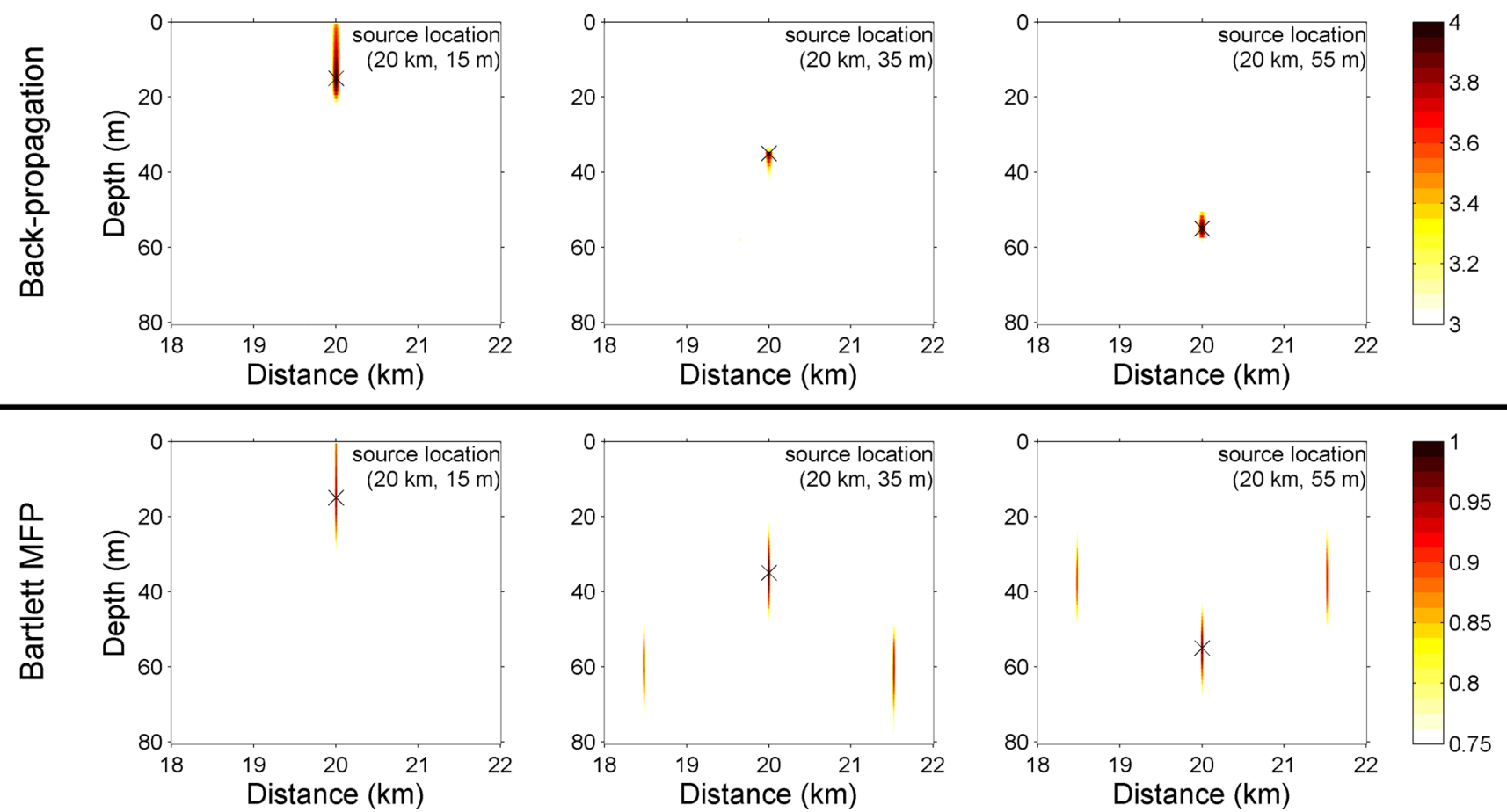

FIG. 2. (Color online) Ambiguity plots of the source location estimate using (top) the normal mode back-propagation approach with four modes and (bottom) the Bartlett MFP. The SNR is $20 \mathrm{~dB}$, and the methods are tested for three different source depths. The color range for plotting is from the maximal possible output to the three-fourths of its value.

approach, its performance under this very low SNR is still considerably good, and not much degradation is observed.

Figure 4 shows the ambiguity plots of source location estimate from the back-propagation localization and the MVDR MFP at $-10 \mathrm{~dB}$ SNR. Even though there is a peak at the true source location in the MVDR MFP, the shallow depth region is saturated with very high values. This makes the localization scheme unable to track the peak without prior information about the source location. The backpropagation ambiguity plot reveals a degradation of the peak height at the true source location, and the side lobes become more noticeable. Nevertheless, the localization performance is still better than the other methods. (a)

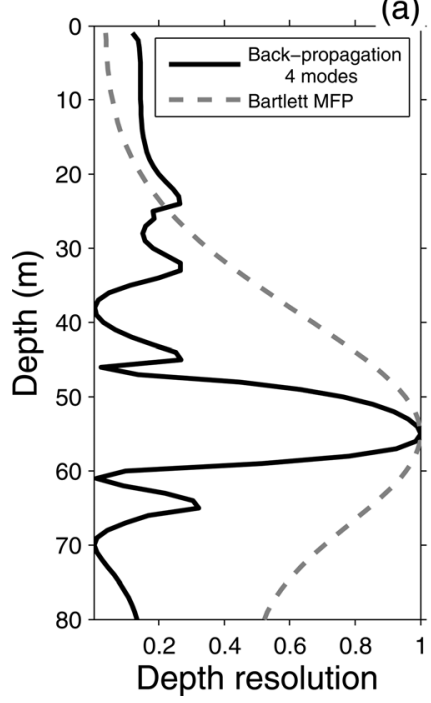

(b)

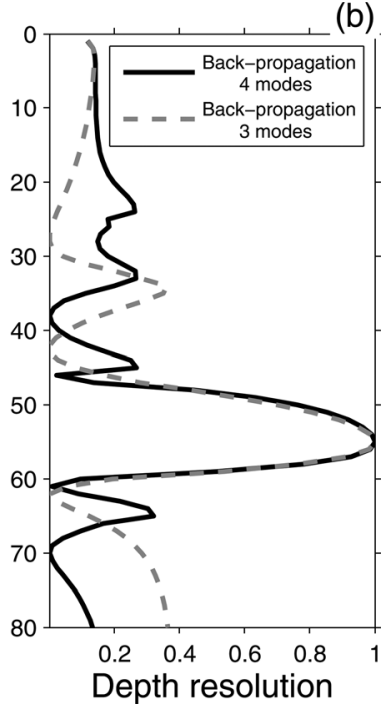

FIG. 3. Depth resolution kernels of different localization schemes.
TABLE I. Noise tolerance comparisons. The source range is at $20 \mathrm{~km}$, and three different source depths are considered. Each entry represents the mean and standard deviation of 50 localizations with a given SNR (20, 10, 0 or $-10 \mathrm{~dB}$ ). SR stands for source range, and SD stands for source depth.

\begin{tabular}{|c|c|c|c|c|c|}
\hline & SNR & $20 \mathrm{~dB}$ & $10 \mathrm{~dB}$ & $0 \mathrm{~dB}$ & $-10 \mathrm{~dB}$ \\
\hline & $\begin{array}{l}\text { SR } \\
\text { SD }\end{array}$ & $\begin{array}{c}\text { mean/std } \\
(\mathrm{km} / \mathrm{m}) \\
(\mathrm{m} / \mathrm{m})\end{array}$ & $\begin{array}{c}\text { mean/std } \\
(\mathrm{km} / \mathrm{m}) \\
(\mathrm{m} / \mathrm{m})\end{array}$ & $\begin{array}{c}\text { mean/std } \\
(\mathrm{km} / \mathrm{m}) \\
(\mathrm{m} / \mathrm{m})\end{array}$ & $\begin{array}{c}\text { mean/std } \\
(\mathrm{km} / \mathrm{m}) \\
(\mathrm{m} / \mathrm{m})\end{array}$ \\
\hline \multicolumn{6}{|c|}{ True source depth $15 \mathrm{~m}$} \\
\hline \multirow{2}{*}{ Back propagation } & $\mathrm{SR}$ & $20.00 / 0.0$ & $20.00 / 0.0$ & $20.00 / 0.0$ & $20.00 / 0.0$ \\
\hline & $\mathrm{SD}$ & $15.00 / 0.0$ & $16.00 / 0.0$ & $17.00 / 0.0$ & $14.68 / 0.5$ \\
\hline \multirow{2}{*}{ Bartlett MFP } & SR & $20.00 / 0.0$ & $20.00 / 0.0$ & $20.00 / 0.0$ & $20.00 / 3.2$ \\
\hline & SD & $15.00 / 0.0$ & $15.02 / 0.3$ & $15.02 / 1.0$ & $14.94 / 3.1$ \\
\hline \multirow{2}{*}{ MVDR MFP } & SR & $20.00 / 0.0$ & $20.00 / 0.0$ & $20.16 / 451.1$ & $20.17 / 489.5$ \\
\hline & $\mathrm{SD}$ & $15.00 / 0.0$ & $12.48 / 5.4$ & $1.28 / 2.0$ & $1.00 / 0.0$ \\
\hline \multicolumn{6}{|c|}{ True source depth $35 \mathrm{~m}$} \\
\hline \multirow{2}{*}{ Back propagation } & $\mathrm{SR}$ & $20.00 / 0.0$ & $20.00 / 0.0$ & $20.00 / 0.0$ & $19.99 / 0.0$ \\
\hline & $\mathrm{SD}$ & $35.00 / 0.0$ & $34.96 / 0.2$ & $35.84 / 0.4$ & $37.40 / 0.5$ \\
\hline \multirow{2}{*}{ Bartlett MFP } & $\mathrm{SR}$ & $20.00 / 0.0$ & $20.00 / 0.0$ & $20.00 / 0.0$ & $20.03 / 937.5$ \\
\hline & SD & $35.00 / 0.0$ & $35.02 / 0.1$ & $34.94 / 0.7$ & $44.84 / 12.5$ \\
\hline \multirow{2}{*}{ MVDR MFP } & SR & $20.00 / 0.0$ & $20.03 / 284.0$ & $20.91 / 914.6$ & $20.73 / 1032.0$ \\
\hline & $\mathrm{SD}$ & $35.00 / 0.0$ & $32.96 / 8.1$ & $1.00 / 0.0$ & $1.00 / 0.0$ \\
\hline \multicolumn{6}{|c|}{ True source depth $55 \mathrm{~m}$} \\
\hline \multirow{2}{*}{ Back propagation } & $\mathrm{SR}$ & $20.00 / 0.0$ & $20.00 / 0.0$ & $20.00 / 0.0$ & $19.99 / 0.0$ \\
\hline & $\mathrm{SD}$ & $55.00 / 0.0$ & $55.00 / 0.0$ & $56.00 / 0.0$ & $53.00 / 0.0$ \\
\hline \multirow{2}{*}{ Bartlett MFP } & SR & $20.00 / 0.0$ & $20.00 / 0.0$ & $20.00 / 0.0$ & $20.06 / 524.2$ \\
\hline & $\mathrm{SD}$ & $55.00 / 0.0$ & $54.98 / 0.1$ & $54.98 / 0.5$ & $52.56 / 5.8$ \\
\hline \multirow{2}{*}{ MVDR MFP } & $\mathrm{SR}$ & $20.00 / 0.0$ & $20.25 / 579.7$ & $20.78 / 1075.5$ & $20.92 / 895.1$ \\
\hline & $\mathrm{SD}$ & $55.00 / 0.0$ & $46.36 / 19.8$ & $1.00 / 0.0$ & $1.00 / 0.0$ \\
\hline
\end{tabular}




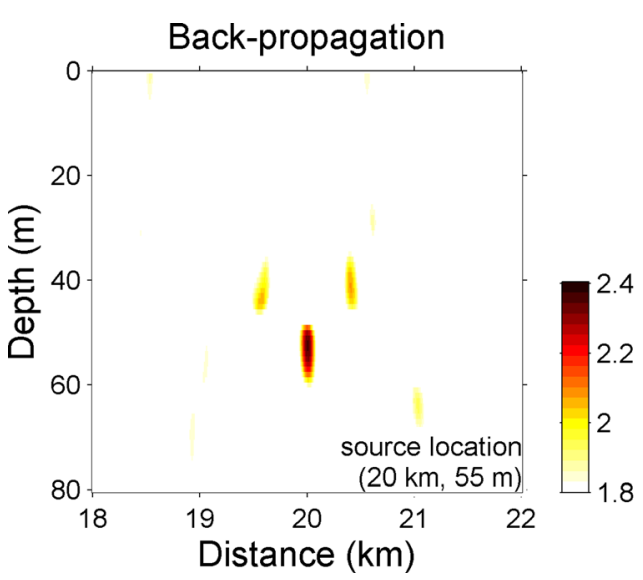

\section{Effects of array tilt}

This example is to examine the tolerance to array tilt. A $20 \mathrm{~dB}$ SNR is considered here. The numerical results show that the MVDR MFP is unable to locate the source. As for the back-propagation and Bartlett MFP methods, their localization performance is summarized in Table II. Three different tilt angles, $5^{\circ}, 3^{\circ}$, and $1^{\circ}$, are considered along with three source locations. The Bartlett MFP performs better (very robust) and tolerates more tilt than the back-propagation localization. The source range estimates from the backpropagation are still accurate. The problem for the backpropagation localization occurs in estimating the depths of shallow sources. The only case where the back-propagation completely fails to locate the source depth is the $5^{\circ}$ tilt and $15 \mathrm{~m}$ source depth. In other cases, the bias of the source depth estimate is still acceptable when comparing to a $100 \mathrm{~Hz}$ wavelength.

TABLE II. Array tilt tolerance comparisons. The source range is at $20 \mathrm{~km}$, and three different tilt angle are considered. Each entry has the mean and standard deviation of 50 localizations. SR stands for source range, and SD stands for source depth. The SNR is $20 \mathrm{~dB}$.

\begin{tabular}{|c|c|c|c|c|}
\hline & Tilt angle & $5^{\circ}$ & $3^{\circ}$ & $1^{\circ}$ \\
\hline & $\begin{array}{l}\text { SR } \\
\text { SD }\end{array}$ & $\begin{array}{c}\text { mean/std } \\
(\mathrm{km} / \mathrm{m}) \\
(\mathrm{m} / \mathrm{m})\end{array}$ & $\begin{array}{c}\text { mean/std } \\
(\mathrm{km} / \mathrm{m}) \\
(\mathrm{m} / \mathrm{m})\end{array}$ & $\begin{array}{c}\text { mean/std } \\
(\mathrm{km} / \mathrm{m}) \\
(\mathrm{m} / \mathrm{m})\end{array}$ \\
\hline \multicolumn{5}{|c|}{ True source depth $15 \mathrm{~m}$} \\
\hline \multirow{2}{*}{ Back propagation } & SR & $20.01 / 0.0$ & $20.00 / 0.0$ & $20.00 / 0.0$ \\
\hline & SD & $1.00 / 0.0$ & $9.00 / 0.0$ & $14.00 / 0.0$ \\
\hline \multirow{2}{*}{ Bartlett MFP } & SR & $20.00 / 0.0$ & $20.00 / 0.0$ & $20.00 / 0.0$ \\
\hline & SD & $16.98 / 0.1$ & $15.78 / 0.4$ & $15.00 / 0.0$ \\
\hline \multicolumn{5}{|c|}{ True source depth $35 \mathrm{~m}$} \\
\hline \multirow{2}{*}{ Back propagation } & SR & $19.88 / 0.0$ & $20.00 / 0.0$ & $20.00 / 0.0$ \\
\hline & SD & $19.00 / 0.0$ & $40.00 / 0.0$ & $36.00 / 0.0$ \\
\hline \multirow{2}{*}{ Bartlett MFP } & SR & $20.00 / 0.0$ & $20.00 / 0.0$ & $20.00 / 0.0$ \\
\hline & SD & $36.00 / 0.0$ & $35.00 / 0.0$ & $35.00 / 0.0$ \\
\hline \multicolumn{5}{|c|}{ True source depth $55 \mathrm{~m}$} \\
\hline \multirow{2}{*}{ Back propagation } & SR & $20.00 / 0.0$ & $20.00 / 0.0$ & $20.00 / 0.0$ \\
\hline & SD & $54.00 / 0.0$ & $55.00 / 0.0$ & $55.00 / 0.0$ \\
\hline \multirow{2}{*}{ Bartlett MFP } & SR & $20.00 / 0.0$ & $20.00 / 0.0$ & $20.00 / 0.0$ \\
\hline & SD & $54.04 / 0.2$ & $55.00 / 0.0$ & $55.00 / 0.0$ \\
\hline
\end{tabular}

FIG. 4. (Color online) Ambiguity plots of the source location estimate with $-10 \mathrm{~dB}$ SNR using (left) the normal mode back-propagation approach with four modes and (right) the MVDR MFP. The color range for plotting is from the maximal output to the three-fourths of its value for the back-propagation localization, and from 0 to 1 for the MVDR MFP.

\section{E. Effects of nonlinear internal waves}

The last numerical example is to test the back-propagation method in the presence of nonlinear internal waves. Figure 5(a) shows the waves of depression considered in this example. This wave train is centered at $10 \mathrm{~km}$ from the source and consists of three ideal squared hyperbolic secant waves with amplitudes equal to $9 \mathrm{~m}, 7.2 \mathrm{~m}$ and $5.4 \mathrm{~m}$, respectively. The themocline of the background sound speed profile shown in Fig. 1(a) is perturbed by the waves, which will cause acoustic mode coupling (Duda and Preisig, 1999). Figure 5(b) presents the modal arrival pulses received on the array located $20 \mathrm{~km}$ away from a $55 \mathrm{~m}$ deep source. The modal arrivals with and without the presence of internal waves are both plotted for comparison, and significant time spreading caused by the mode coupling is seen on modes 1 and 2 .

In this example, four modal arrivals are back-propagated without considering internal waves, as if we do not know their presence. The ambiguity plot of source location estimates is shown in Fig. 5(c), which has multiple peaks caused by the mode coupling. Note that the coupled (scattered) modal arrivals are back-propagated adiabatically. So, these coupled modes may correlate to each other at multiple locations, but the correlation value will be weaker than the case when there are no internal waves. This is seen in Fig. 5(c), where the peak value is lower than the one shown in the upper right panel of Fig. 2.

The Bartlett MFP is also tested, and the signal replica is calculated without considering internal waves. The pattern of the Bartlett MFP ambiguity plot [Fig. 5(d)] remains the same as the case where there are no internal waves (the lower right panel of Fig. 2), but has lower peak values. Comparing the ambiguity plots of the back-propagation and Bartlett MFP methods, we still see that the back-propagation performs better since it has smaller side-lobe deviation from the true location.

\section{APPLICATIONS TO SW06 EXPERIMENTAL DATA}

Two source localization examples using the Shallow Water 2006 (SW06) experiment data are presented in this section. A variety of environmental measurements made 
(a) Nonlinear internal waves (NIW)

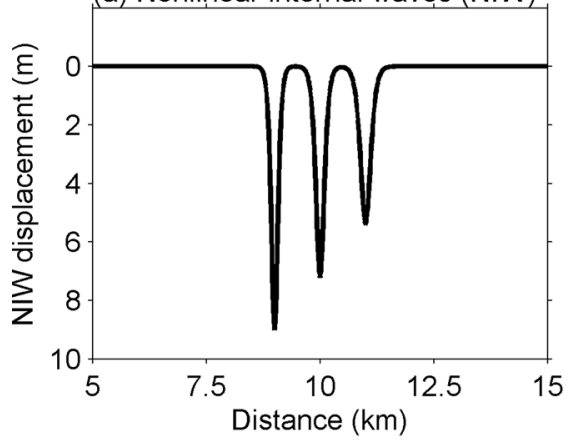

(b) First three acoustic modal arrivals

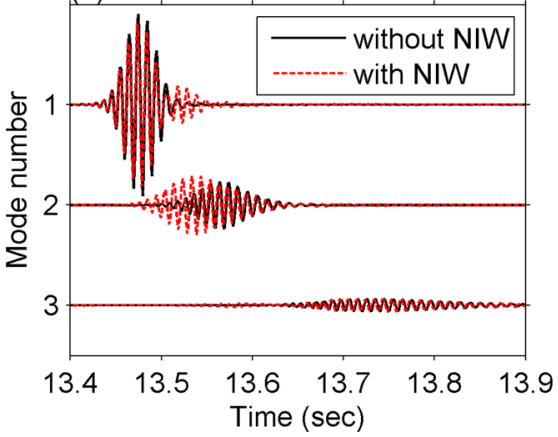

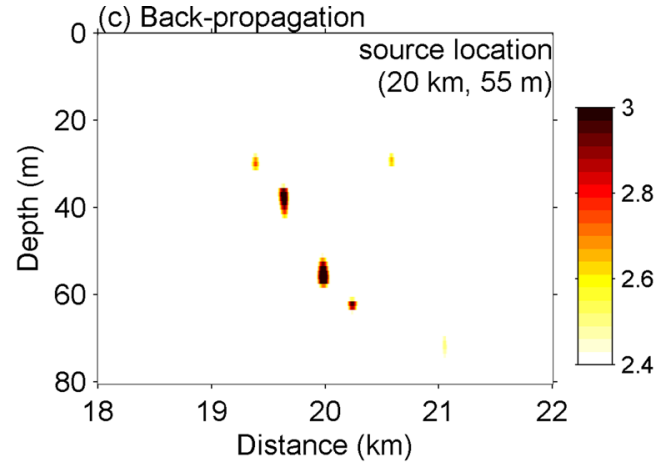

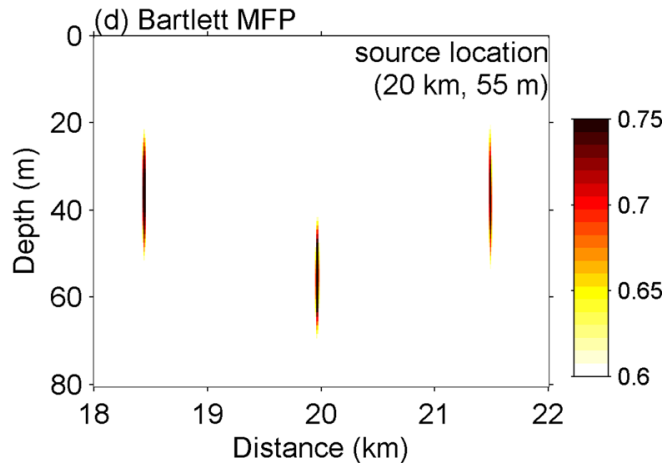

FIG. 5. (Color online) Source localization in the presence of nonlinear internal waves. (a) The wave form used in the example to perturb the themocline of the background sound speed profile. (b) The arrivals of first three modes received on the hydrophone array located $20 \mathrm{~km}$ away from the source. (c and d) The ambiguity plots of source location estimates from back-propagation and Bartlett MFP, respectively. during this experiment are used to achieve better performance for source localization. These measurements include sound speed profiles, barotropic and baroclinic tides, bottom topography and sub-bottom geoacoustic properties, which are reviewed below.

\section{A. SW06 environmental measurements}

The SW06 experiment was a large interdisciplinary experiment conducted on the edge of the continental shelf off New Jersey during the summer of 2006 (Tang et al., 2007). This experiment had two main components for studying acoustics and oceanography in a shelfbreak region. Figure 6 shows locations of the moorings from which the acoustic and oceanographic data used in this paper were collected. Environmental measurements were made on the moorings ENV\#32 and ENV\#45. The mooring ENV\#45 was equipped with temperature sensors, and the mooring ENV\#32 had temperature, conductivity and pressure sensors. The acoustic receiving system is labeled "WHOI array" as it is owned and operated by Woods Hole Oceanographic Institution (WHOI). The system had 16 hydrophones covering three-fourths of the water column ( $\sim 80 \mathrm{~m}$ deep) from $13 \mathrm{~m}$ to $75 \mathrm{~m}$ in depth and forming a vertical line array (VLA). Additionally, there were 32 hydrophones spaced at $15-\mathrm{m}$ intervals forming a horizontal line array (HLA) on the bottom and directed to the north. Both the VLA and HLA were navigated by an acoustic long baseline (LBL) system (Newhall et al., 2007), and the navigation results are incorporated in the source localizations presented below. The WHOI VLA also had temperature sensors whose data are used in this paper. Two acoustic sources, the Miami Sound Machine (MSM, owned and operated by University of Miami) and a J-15-3 source operated by Penn State University and WHOI, will be used for testing the localization methods.
The sound speed measurements, derived from temperature and salinity data, are shown in Figs. 7(a)-7(c). They reveal the complicated frontal variability known at this location, including the foot of the front, frontal intrusions in the middle of the water column and internal waves and tides. Each of these features can cause sound speed variability, and a paper by Colosi et al. (2012) is referred to for detailed discussions. The barotropic tides, shown in Fig. 7(d), are M2 dominant, and this record is used to determine the timevarying water depth in the localization procedure. Detailed bathymetry and sub-bottom structure are also used, as shown in Fig. 7(e). The bathymetry data is from the 1996 STRATAFORM swath map survey, and the sub-bottom geoacoustic model is obtained from a previous study (Ballard et al., 2010).

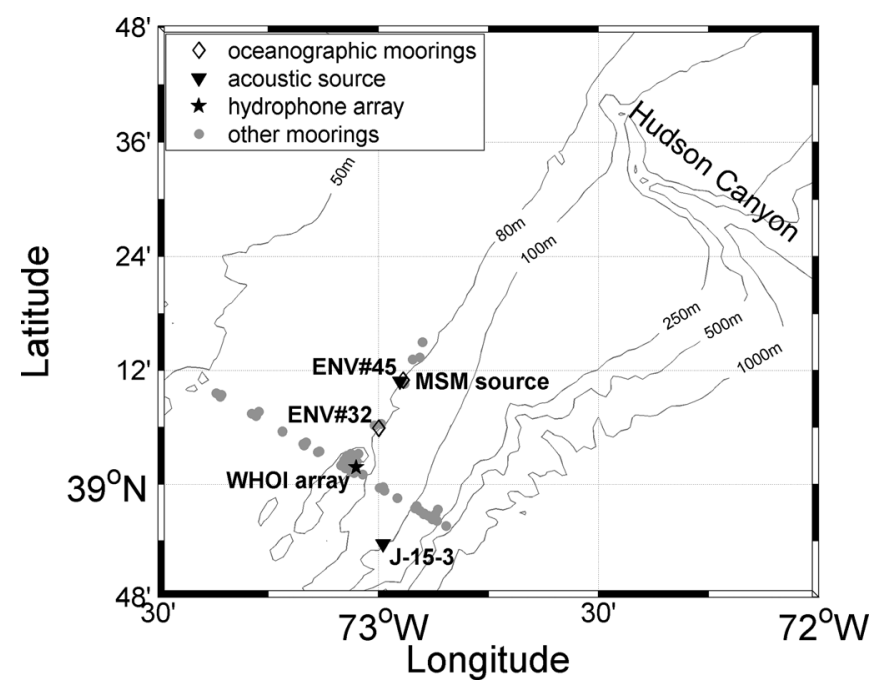

FIG. 6. SW06 experimental area and mooring locations.

Lin et al.: Source localization using back-propagation 

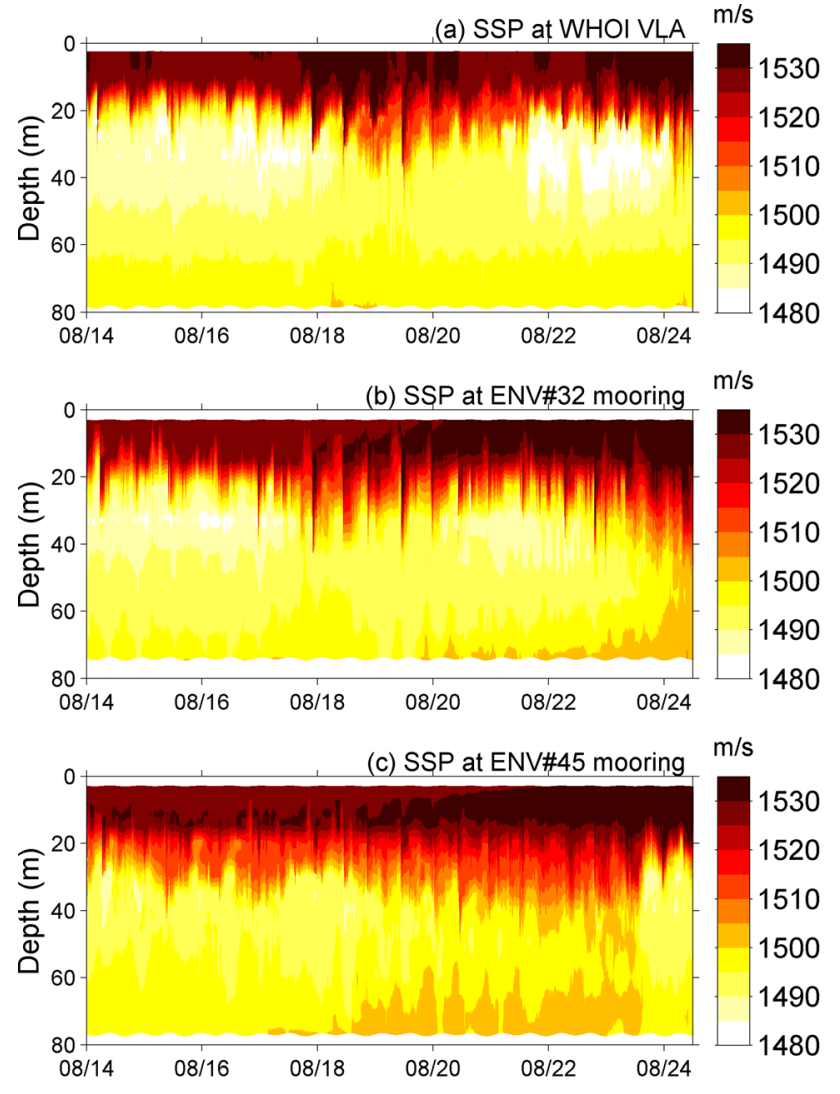
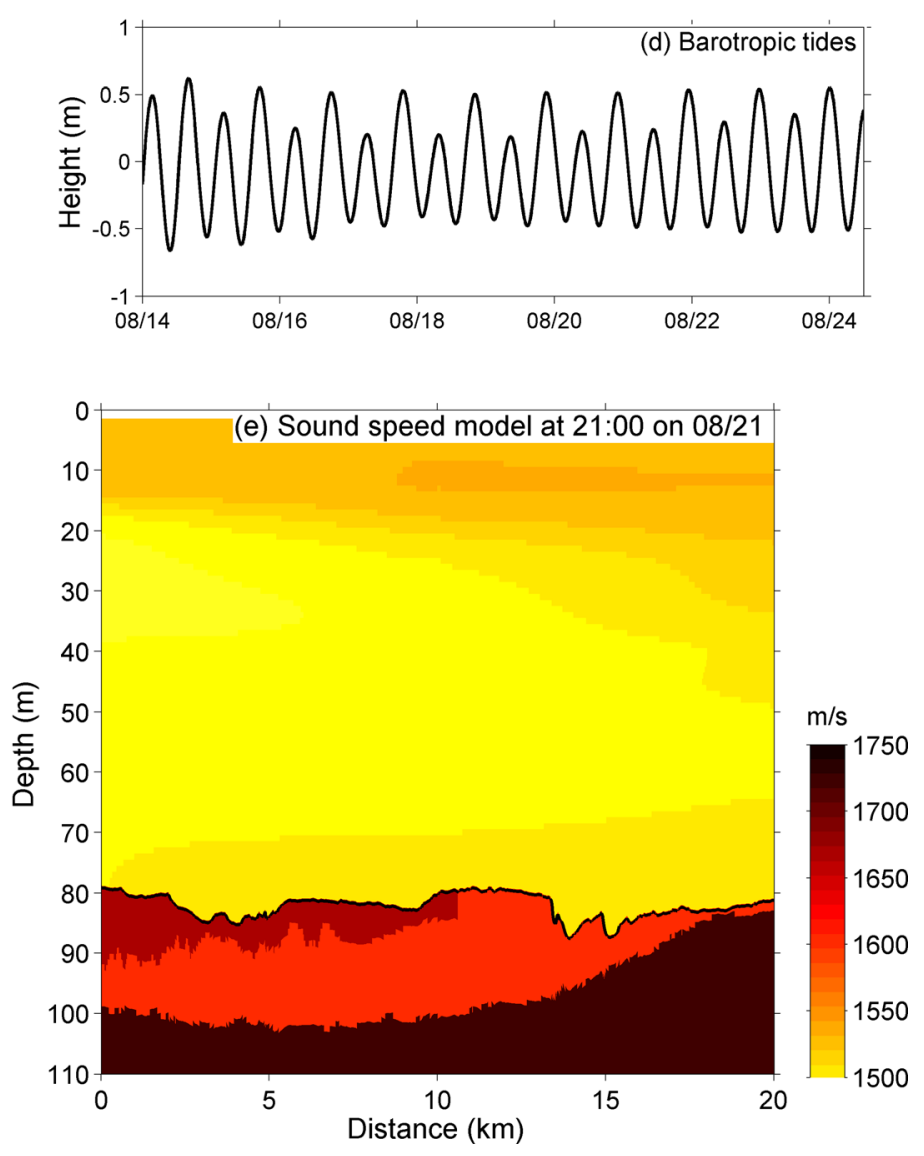

FIG. 7. (Color online) Environmental measurements used for localizing acoustic sources in the SW06 experiment. (a)-(c) Water sound speed measurements, and (d) the record of barotropic tides. Data from August 14 to 24 are shown. (e) The sound speed model used in source localization at one time. This model includes detailed bathymetry and sub-bottom structure, and the water sound speed varies with time (e.g., the baroclinic internal tides show clearly).

\section{B. MSM sound source localization}

Back-propagation localization of the moored MSM source is presented next. The source was positioned at $56 \mathrm{~m}$ depth and $19.74 \mathrm{~km}$ northeast $\left(25.73^{\circ}\right.$ due north) from the WHOI VLA. It transmitted M-sequence phase encoded signals in 5 different frequency bands $(100 \mathrm{~Hz}, 200 \mathrm{~Hz}, 400 \mathrm{~Hz}$, $800 \mathrm{~Hz}$, and $1600 \mathrm{~Hz}$ ), and the $100 \mathrm{~Hz}$ signal (with a $25 \mathrm{~Hz}$ bandwidth) is analyzed here. Every half hour, a 1.5-min long transmission, which contained 36 identical M-sequence phase encoded signals, was emitted. Received signals from August 14 to 24 are considered here, and source localization is done without employing pulse compression. The source bearing from the WHOI VLA is given in this example, and four modes will be back-propagated along the known source bearing to determine the source location and the source depth.

\section{Vertical Mode filtering}

Mode filtering with the WHOI VLA requires local mode functions, and these were calculated using sound speed profile time series and a bottom model obtained from previous studies (Lin et al., 2010; Ballard et al., 2010). A Gauss Markov mode filter is employed, and the detailed procedure is described below.

For each MSM signal, the noise covariance $\hat{\mathbf{R}}_{n n}$ is estimated from the noise records taken right after the MSM transmission period at the VLA, and the signal covariance
$\hat{\mathbf{R}}_{A A}$ is estimated by a pseudo-inverse mode filter using data taken within a six-hour time window. This is similar to what has been done in the previous numerical examples, and the signal variations in the six-hour window (half of the M2 tide period) and the noise variations in the 3-min window are assumed to be weak-sense stationary. The method of replacing zero eigenvalues with the minimal non-zero eigenvalue is used to regulate the sampled $\hat{\mathbf{R}}_{n n}$ and $\hat{\mathbf{R}}_{A A}$. After the signal is mode-filtered, the residue $\hat{\mathbf{n}}$ of the Gauss-Markov mode filter is calculated from

$$
\hat{\mathbf{n}}_{\mathrm{N} \times 1}=\mathbf{P}_{\mathrm{N} \times 1}-\boldsymbol{\Psi}_{\mathrm{N} \times \mathrm{M}} \hat{\mathbf{A}}_{\mathrm{M} \times 1},
$$

where the variables are defined in Eq. (9). When the statistics of the filter residues are consistent with the noise measurements, the Gauss-Markov mode filter can be considered as only extracting the signal.

It is found that the a priori noise information is more crucial for successful noise-signal separation. The spatialspectral cross-correlation is used in the Gauss-Markov mode filter, and the use of a three-minute noise window is adequate for tracking the noise variations in the SW06 data. A priori statistics $\hat{\mathbf{R}}_{A A}$ and $\hat{\mathbf{R}}_{n n}$ will be used in the GaussMarkov mode filter to produce a posteriori uncertainty for the modal amplitude estimates. Within the framework of the adaptive normal-mode back-propagation, the posteriori uncertainty is then used in the implementation of back- 
propagation inversion, which is formulated in Eq. (6) and explained previously in Sec. III B.

One example of mode filtering is illustrated in Fig. 8 to show the performance of the Gauss Markov mode filter with noisy data recorded around 11:30 (UTC) on August 22. Figure 8 (a) shows the spectrogram of three minutes of data at depth $28.5 \mathrm{~m}$, and only the first $1.5 \mathrm{~min}$ contain MSM signals. The Gauss Markov mode filter is performed to extract those signals, and the filter residues at depth $28.5 \mathrm{~m}$ are shown in Fig. 8(b). The statistics of the residues are compared to the noise measurements in the second 1.5-min data, and they agree with each other very well, which means the Gauss-Markov mode filter only extracted the signal. This is expected since the spectral pattern of the filter residues and the noise data is similar, as shown in Fig. 8(b). To demonstrate the advantage of our approach, the pseudo-inverse mode filter without considering the statistics of signal and noise is also performed, and the filter residues are plotted in Fig. 8(c). One can see that, unlike the Gauss-Markov mode filter, the pseudo-inverse mode filter passes both signal and noise, and only small amount of residue remains. The modal arrivals extracted for one MSM signal using both methods are plotted in Fig. 9. Note that pulse compression is performed here to visualize the signal, and the
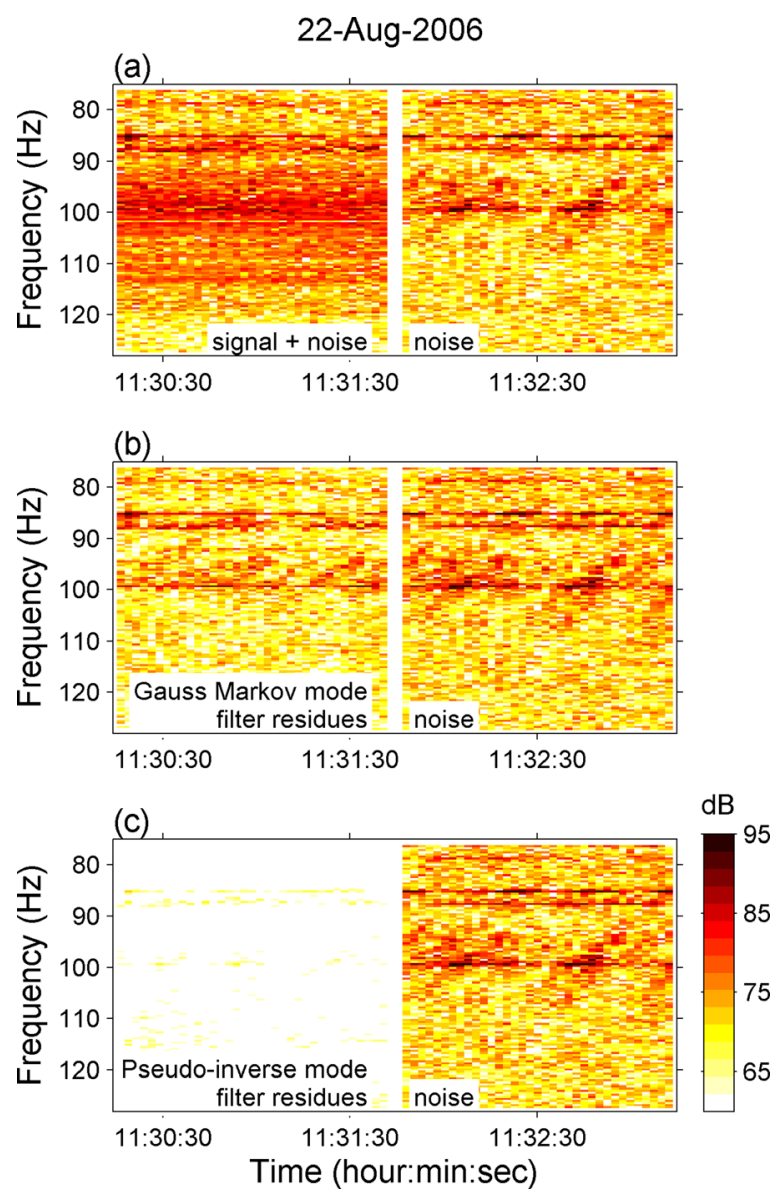

FIG. 8. (Color online) (a) The spectrogram of the received MSM signals at $28.5 \mathrm{~m}$ depth around 11:30 (UTC) on August 22, 2006. The first 1.5-min data contains both signal and noise, and the second 1.5-min data contains only noise. (b) The Gauss-Markov mode filter is carried out with noise covariance estimates obtained from the noise, and the filter residues are shown. (c) The pseudo-inverse mode filter is also carried out for a comparison, and the filter residues are shown.
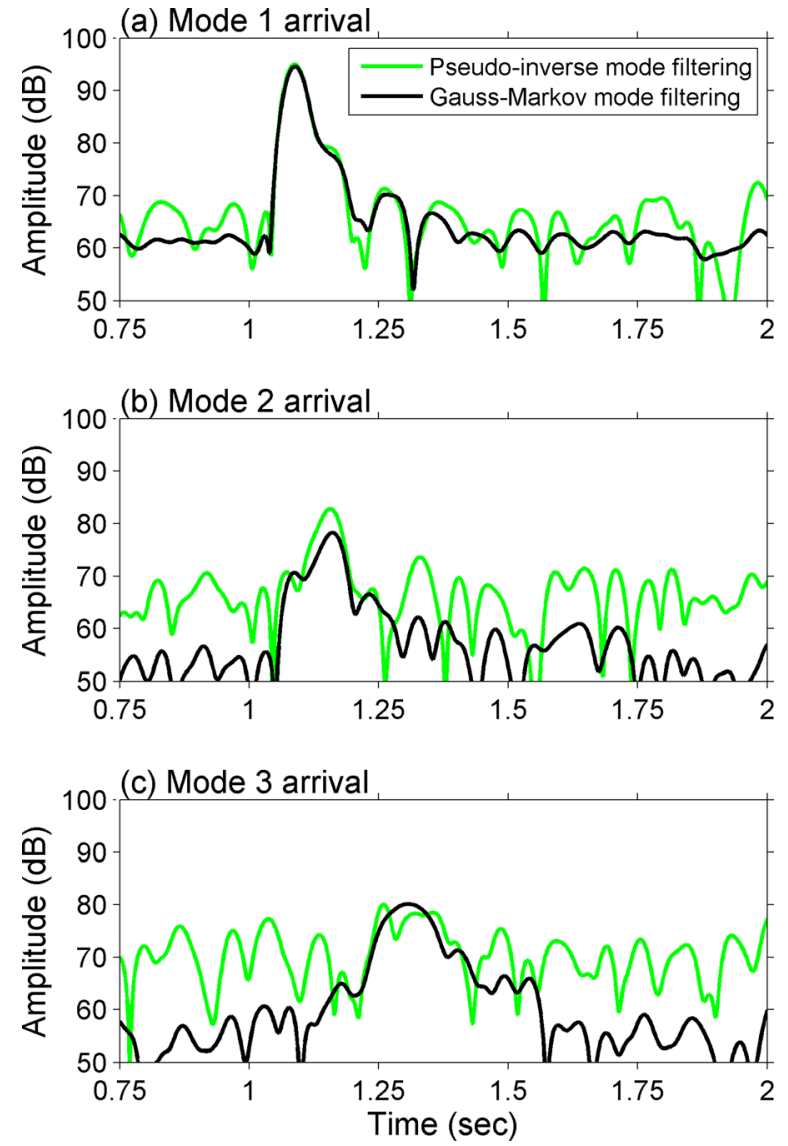

FIG. 9. (Color online) Mode filtering comparisons. The data is shown in the third column of Fig. 8, and it is clear that the Gauss-Markov mode filter rejects the noise and preserves the signal nicely.

Gauss-Markov mode filter indeed rejects the noise and preserves the signal nicely. This is important because we do not want to back-propagate the noise along with the signal when we are performing the source localization.

\section{Normal mode back-propagation and source localization results}

Two dimensional in-plane propagation is assumed here, and the back-propagation does not consider mode-coupling, which is responsible for some error in the localization results. The sound speed field along the acoustic track is calculated with a spatial interpolation procedure using sound speed data measured at three moorings, WHOI VLA, ENV\#32 and ENV \#45 (see Fig. 7(e) for an example of the sound speed model). Accurate bathymetry and barotropic tide records were used to produce water depth in the model. A detailed sub-bottom model was also used. Four modes are calculated every $150 \mathrm{~m}$ and back-propagated in $25 \mathrm{~m}$ steps.

Ten days of MSM data are processed. Every half hour, 35 pulses within $1.5 \mathrm{~min}$ are analyzed, and 35 source range and depth estimates are obtained. The distributions of localization results are shown in Fig. 10. The total mean range estimate is $19.77 \mathrm{~km}$, very close to the true distance $(19.74 \mathrm{~km})$, along with a standard deviation of $950 \mathrm{~m}$. The depth estimates reveal a bi-modal distribution at $56 \mathrm{~m}$ and $42 \mathrm{~m}$, which is most likely due to the mode-coupling that we do not account 


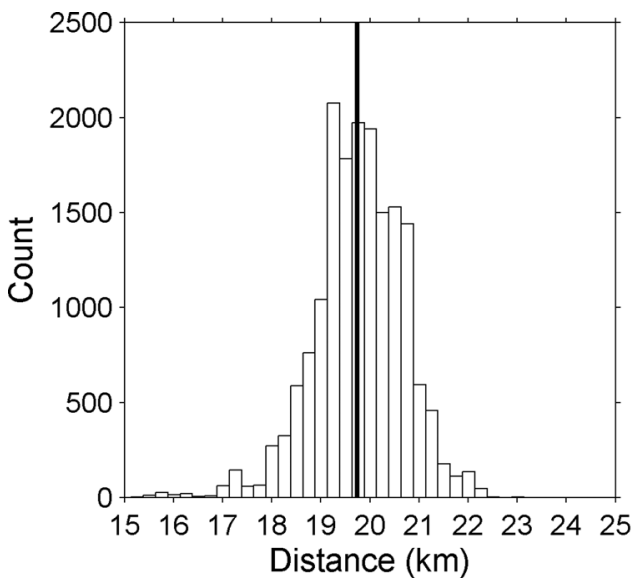

for in the back-propagation, as described by the numerical example shown in Sec. IV E. Note that the higher peak of the bi-modal distribution is at the true source depth $(56 \mathrm{~m})$.

\section{Environmental effects}

Some environmental effects are significant in the MSM source localization. The average value and standard deviation of the range estimates in each transmission period $(1.5 \mathrm{~min})$ are plotted in Figs. 11(a) and 11(b), along with nonlinear internal wave signals plotted in Fig. 11(c). It can be observed that at the times when internal waves were present, the standard deviation of the $1.5 \mathrm{~min}$ localization record also increased. It is known that nonlinear internal waves can distort the coherent structure of the sound field due to mode coupling (Duda and Preisig, 1999) and 3-D sound propagation effects (Lynch et al., 2010). When there is a nonlinear internal wave group in the propagation path, the localization suffers from these propagation effects and the performance can be degraded.

Sub-mesoscale variability is also considerable in this experimental area since the experiment was located at the edge of continental shelf and subject to the variability of the shelf-break front. This can be seen in Figs. 7(a)-7(c). Note that the water column mooring measurements were separated by $\sim 10 \mathrm{~km}$. The spatial Nyquist sampling rate of these measurements is thus $20 \mathrm{~km}$, meaning the water-column variability with a wavelength less than $20 \mathrm{~km}$ along the propagation path is not resolvable.

Another environmental effect could be from the subbottom acoustic properties. From the non-biased mean in the source range estimates, the sound speed structure, including sub-bottom layering, used in the environmental model is believed to be quite accurate. However, the attenuation coefficients in the bottom are not well understood, and this can explained why the cost function of the back-propagation localization only reaches about 2.4 , not the expected maximal value, which should be 4 since four modes are used. In fact, the back-propagated modes do coincide with each other in time, but the compensation for modal attenuation is not perfect so that the cost function can not reach its maximal value.

\section{J-15-3 sound source localization}

Signals collected from a broadband experiment conducted by Penn State University (Rajan and Becker, 2010) during the SW06 experiment are also used to test the backpropagation approach. The signals were linear chirps with a bandwidth of $250 \mathrm{~Hz}(40-290 \mathrm{~Hz})$ transmitted from a J-15-3 source and received by the WHOI arrays. The signal duration was $0.5 \mathrm{~s}$, and chirps were transmitted for a certain period at stations circled around the WHOI VLA. One station was selected for testing back-propagation source localization across the shelfbreak. The details of this broadband experiment and its application on geoacoustic inversion are referred to Ballard et al. (2010) and Rajan and Becker (2010). Full 3-D source localization including source bearing, range and depth are performed.

$\mathrm{J}-15-3$ data in a frequency band from $75 \mathrm{~Hz}$ to $125 \mathrm{~Hz}$ is used. The reasons for selecting this band are that (1) it
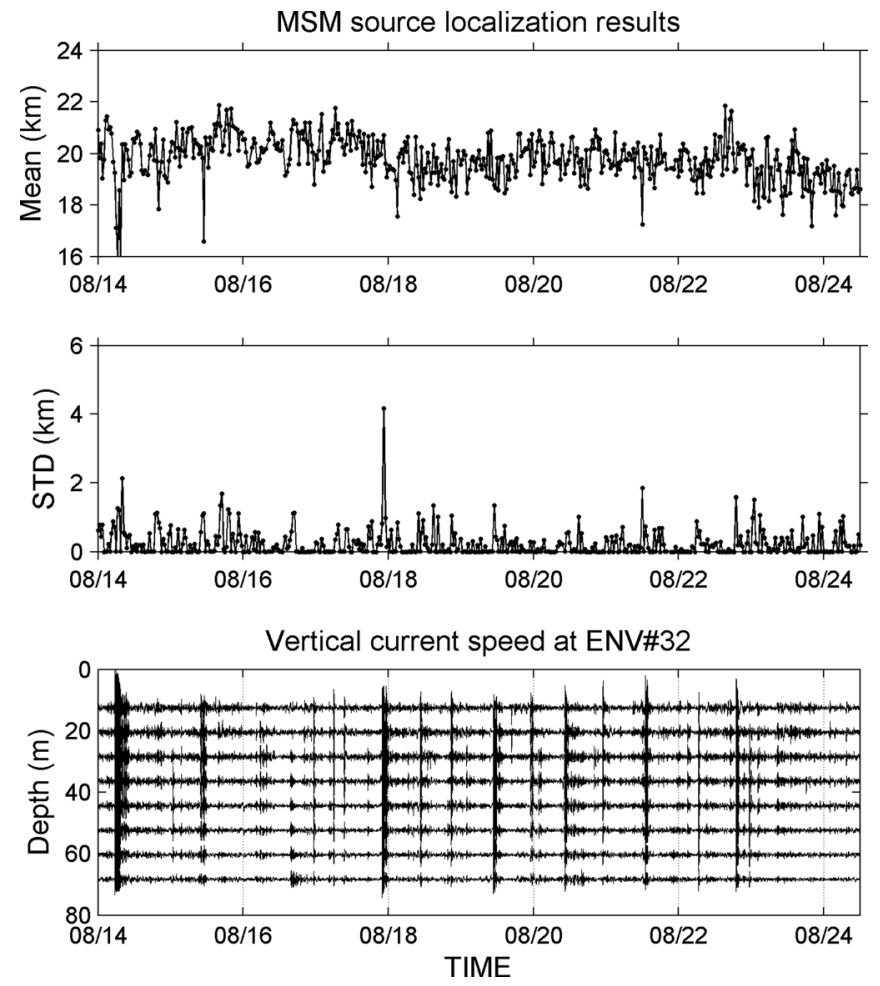

FIG. 11. Effects of nonlinear internal waves on MSM source localization. The vertical current speeds measured at the mooring ENV\#32 are good indicators of nonlinear internal waves. From a statistical hypothesis test, the short-time standard deviations of range estimates are found to be correlated with the internal wave signals. 
coincides with the bandwidth of the MSM $100 \mathrm{~Hz}$ pulse, so a direct comparison with the previous example can be achieved, and (2) strong noise exists in this bandwidth, so the signal/noise separation with the Gauss Markov model filter can be tested. In this example, three modes are used and back-propagated to localize the source.

\section{Vertical mode filtering}

The Gauss-Markov mode filter is employed to analyze the J-15-3 chirp signals. These chirps were transmitted in 3-second intervals. At the J-15-3 station analyzed here, the transmission lasted for $12 \mathrm{~min}$, and a total number of 220 pulses are analyzed, and the second moment of the received signal $\hat{\mathbf{R}}_{A A}$ is estimated from the entire transmitted pulses. To filter each signal, a 12-s center window is utilized to estimate the noise covariance $\hat{\mathbf{R}}_{n n}$ around the signal time. A mode filtering example is shown in Fig. 12, and, as one can see in the estimated modal arrivals, the Gauss-Markov mode filter produces a lower noise floor.

\section{Horizontal array beamforming with normal modes}

The HLA beamforming implemented here is the conventional Bartlett Matched-Field Processing shown in Eq. (13) with the replica field made up for the modal arrivals along the HLA. The replica is calculated using plane-wave normal mode propagation with the mode amplitude estimates at the VLA. The advantage of this beamforming technique is to eliminate the phase speed mismatch which may occur when using the conventional plane wave beamforming in an acoustic waveguide consisting of multiple normal modes.

Figure 13(a) shows the beamforming output in the dotted line and the shipboard Global Position System (GPS) data with the solid line. The bearing angles agree very well but with a $1^{\circ}$ constant bias. Since the WHOI HLA employed a long-base line (LBL) system (Newhall et al., 2007), this $1^{\circ}$ beamforming bias should not be caused by array position errors. Possible causes are discussed below.

One suspect for the beamforming bias is the error in the theoretical calculation of the local modes around the VLA, specifically on the modal phase speeds, which can be caused by the environmental mismatch in the model utilized. The water column sound speeds at the WHOI VLA were obtained from an objective merging process using all physical oceanographic data collected around the VLA (Lin et al., 2010). The local sub-bottom model at the VLA was obtained from an independent geoacoustic inversion with a good confidence level (Ballard et al., 2010). The barotropic tide is also considered for adjusting the water depth at the time when the J-15-3 broadband experiment was conducted. While the environmental mismatch is believed to be small, it may still contribute to the observed $1^{\circ}$ beamforming bias.

Another suspect for this $1^{\circ}$ offset error is 3-D horizontal refraction. As shown in Fig. 6, the propagation path from the J-15-3 station to the WHOI VLA was oblique across the shelfbreak, which may result in horizontal refraction due to a sloping bottom (Doolittle et al., 1988). A detailed analysis is required to confirm what exactly causes the small beamforming bias. Since it is beyond the scope of this paper, we defer it for future research.

\section{Normal mode back-propagation and localization results}

The normal mode approach is employed to localized the $\mathrm{J}-15-3$ source. 2-D in-plane propagation is assumed, and the

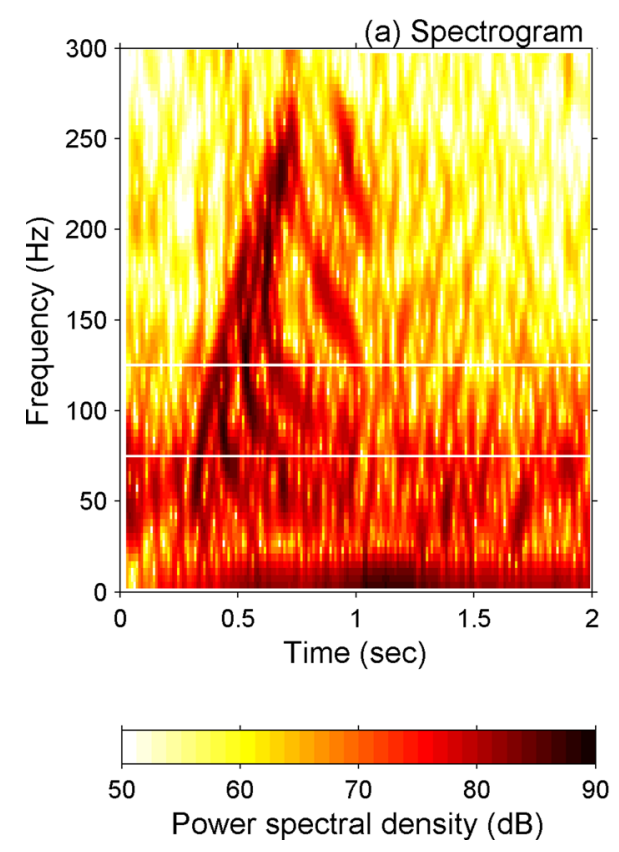

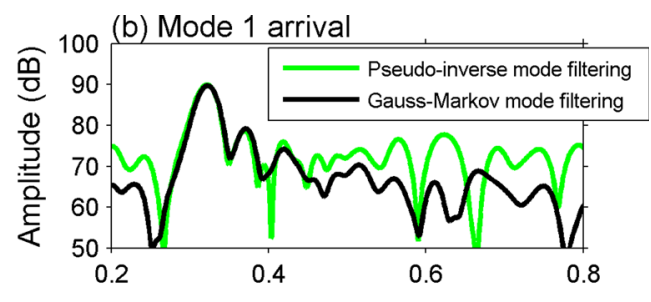
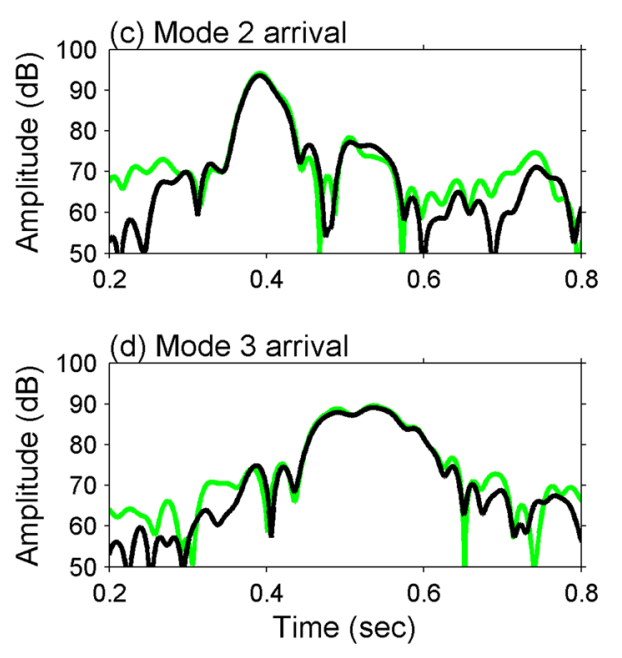

FIG. 12. (Color online) A mode filtering example of J-15-3 signal. (a) Spectrogram of the received signal on the hydrophone at $36 \mathrm{~m}$ depth at 21:59 (UTC) on August 6. The two thin lines mark the frequency band $(75 \mathrm{~Hz}$ to $125 \mathrm{~Hz}$ ) where the mode filters are applied. (b and c) Mode filtering comparisons. Clearly, the Gauss-Markov mode filter rejects the noise nicely. 

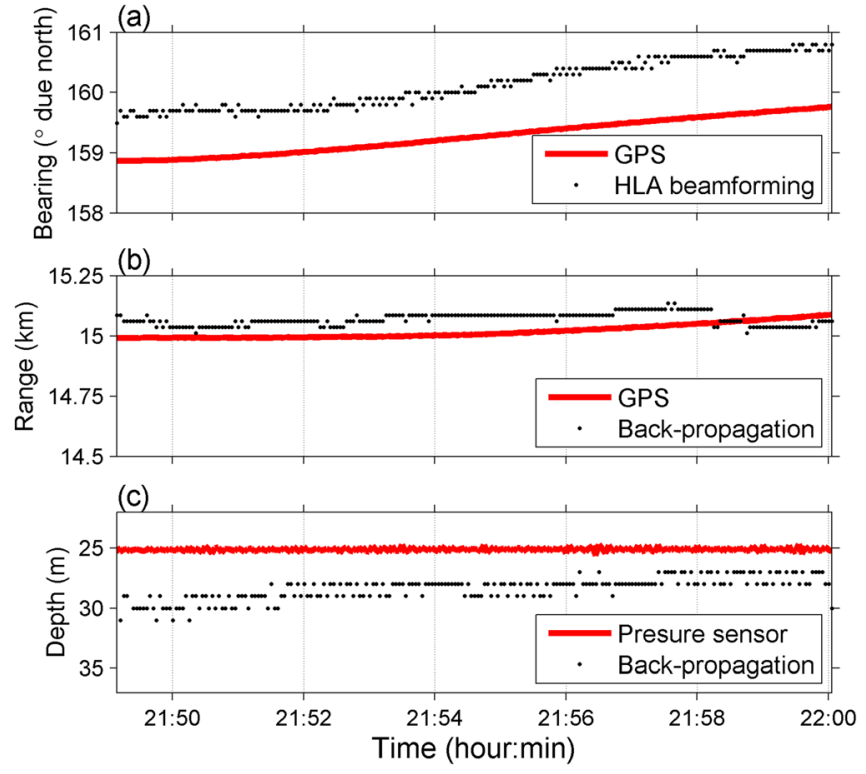

FIG. 13. (Color online) J-15-3 localization results: (a) source bearing, (b) source range, and (c) source depth.

search grid of source range and depth is along the source bearing obtained from the horizontal array beamforming. The environmental model for calculating the theoretical normal modes along the source bearing is constructed differently than the previous MSM example due to the lack of water column and sub-bottom information. The sub-bottom structure model along this path is outside the grid of the detailed geoacoustic study (Ballard et al., 2010; Rajan and Becker, 2010), so a one-layer sub-bottom model is used. The sub-bottom model layer is $150-\mathrm{m}$ thick with a constant sound speed of $1640 \mathrm{~m} / \mathrm{s}$ on the top of a basement with $1740 \mathrm{~m} / \mathrm{s}$ sound speed. The density of the bottom model is $1.9 \mathrm{~g} / \mathrm{cm}^{3}$, and the attenuation coefficient is $0.2 \mathrm{~dB}$ per wavelength. The geoacoustic properties of this bottom model are selected from the average values of the geoacoustic inversions near the area (Ballard et al., 2010; Rajan and Becker, 2010) with some tuning to match the true source range.

Lack of water column measurements is also an issue in this example. Two sound speed profiles at both ends of the track are used to construct two range-independent segments (nearest neighbour interpolation). The sound speed profiles measured at the VLA are used for the receiver side, and a single CTD cast (Rajan and Becker, 2010) conducted immediately after the J-15-3 transmission at the station is used for the source side. One may wonder why we did not use the mooring measurements along the across-shelf mooring line (see Fig. 6). During the SW06 experiment, a fleet of submarine gliders were deployed to measure the sub-mesoscle oceanographic variability in the experiment area. It is found that the along-shelf variability is complicated by the shelfbreak front process. In fact, the glider data collected during the J-15-3 experiment shows that the along-shelf variability was so intense that the spatial correlation range was limited (Gong et al., 2008), and the simple extrapolation of the data along the across-shelf mooring line to the propagation path of the J-15-3 signal track is questionable. A comparison of the J-15-3 CTD profile with the mooring data is made, and the de-correlation agrees with the physical oceanographic picture drawn from the glider observations: the frontal intrusions and filaments were across the area of J-15-3 station at the time, and changed the structure of the mid-water column sound speed field. We shall certainly bear in mind this environmental variability, along with the uncertain sub-bottom structure, in the examination of the back-propagation source localization.

Figures 13(b)-13(c) present the results from the backpropagation source localization. First, in Fig. 13(b), the source range estimates agree with the shipboard GPS data well. Note that the source was slightly drifting during the transmission. Some bias of $\sim 100 \mathrm{~m}$ is observed before 21:58, and the bias becomes less afterward. Although some adjustment to the geoacoustic parameters may improve the overall agreement, it will only shift the entire localization results up or down, and the jump at 21:58 will still remain. The most plausible reason for this jump may be due to the water column variability which is not accounted for in the model. This might be an interesting point to investigate in the future.

The source depth estimates shown in Fig. 13(c) are satisfactory, as can be seen with a comparison to the ground truth from the pressure sensor on the source. Considering the aspect ratio of the distance to the source and the water depth $(15 \mathrm{~km}$ to $\sim 100 \mathrm{~m}$ ), the bias of a few meters seen in the depth estimates should be considered small. The environmental mismatch can certainly contribute an error of this order of magnitude. Also, the assumption made in the sound propagation model, i.e., 2-D adiabatic mode propagation, may also be responsible.

\section{CONCLUSIONS}

An adaptive normal mode back-propagation approach for low-frequency broadband sound source localization from a single station in a shallow-water ocean has been presented. Gauss-Markov inverse theory is used in both mode filtering and back-propagation, and unifies our adaptive backpropagation method. The accuracy and robustness of this method are confirmed via simulation studies and comparisons to other methods.

This adaptive back-propagation method was also successfully applied to localize two distant sources in the SW06 experiment, which demonstrates the feasibility of this method for real-data applications in a shallow water environment. In the first SW06 example, we localized the Miami Sound Machine (MSM) along the shelf and found that nonlinear internal waves are responsible for range estimate deviations over small time-scales ( $<2 \mathrm{~min})$. In a second example, we localized a J-15-3 source across the shelfbreak, and mode coupling due to the sloping bottom and the internal waves were likely to cause some error. Insufficient sub-mesoscale sound speed measurements may also be responsible for errors seen in both examples. Incorporating a regional data-assimilating ocean model for the sub-mesoscale sound speed field is work in progress by the authors and their collaborators.

In theory, the adiabatic mode assumption used in the backpropagation method can be relaxed. The equations presented here can be readily generalized to consider mode coupling by cascading the mode content matrix $\mathbf{E}_{m}$ over mode number and 
simultaneously back-propagating all modes. However, lack of environmental data on the sound speed field (especially the horizontal gradients) along the propagation path will be an insurmountable impediment to including mode coupling for source localization in most all practical applications.

\section{ACKNOWLEDGMENTS}

The SW06 experiment was supported by the Office of Naval Research, and heartfelt thanks must be offered to the acoustics and physical oceanography program managers Dr. Ellen Livingston and Dr. Terry Paluszkiewicz. The authors also thank all of the SW06 experiment participants for their efforts to make this large, multi-disciplinary, shallow-water ocean experiment successful.

Baggeroer, A. B., Kuperman, W. A., and Mikhalevsky, P. N. (1993). "An overview of matched field methods in ocean acoustics," IEEE J. Oceanic Eng. 18, 401-424.

Ballard, M. S., Becker, K. M., and Goff, J. A. (2010). "Geoacoustic inversion for the New Jersey Shelf: 3-D sediment model,” IEEE J. Ocean. Eng. 35, 28-42.

Baumgartner, M. F., Freitag, L., Partan, J., Ball, K. R., and Prada, K. E. (2008). "Tracking large marine predators in three dimensions: The realtime acoustic tracking system,” IEEE J. Ocean. Eng. 33, 146-157.

Buck, J. R., Preisig, J. C., and Wage, K. E. (1998). "A unified framework for mode filtering and the maximum a posteriori mode filter," J. Acoust. Soc. Am. 103, 1813-1824.

Bucker, H. P. (1976). "Use of calculated sound fields and matched-field detection to locate sound sources in shallow water," J. Acoust. Soc. Am. 59, 368-373.

Chiu, C.-S., Miller, C. W., and Lynch, J. F. (1997). "Optimal modal beamforming of bandpass signals using an undersized sparse vertical hydrophone array: Theory and a shallow-water experiment," IEEE J. Ocean. Eng. 22, 522-533.

Clark, C. W., Brown, M. W., and Corkeron, P. (2010). "Visual and acoustic surveys for North Atlantic right whales, Eubalaena glacialis, in Cape Cod Bay, Massachusetts, 2001-2005: Management implications," Mar. Mamm. Sci. 26, 837-854.

Colosi, J. A., Duda, T. F., Lin, Y.-T., Lynch, J. F., Newhall, A. E., and Cornuelle, B. (2012). "Observations of sound-speed fluctuations on the New Jersey continental shelf in the summer of 2006," J. Acoust. Soc. Am. 131, 1733-1748.

Dizaji, R. M., Chapman, N. R., and Kirlin, R. L. (2002). "A phase regulated back wave propagation technique for geoacoustic inversion," J. Acoust. Soc. Am. 111, 800-808.

Doolittle, R., Tolstoy, A., and Buckingham, M. J. (1988). "Experimental confirmation of horizontal refraction from a point source in a wedgeshaped ocean,” J. Acoust. Soc. Am. 83, 2117-2125.

Duda, T. F., and Preisig, J. C. (1999). "A modeling study of acoustic propagation through moving shallow-water solitary wave packets," IEEE J. Oceanic Eng. 24, 16-32.

Ferris, R. H. (1972). "Comparison of measured and calculated normal-mode amplitude functions for acoustic waves in shallow water," J. Acoust. Soc. Am. 52, 981-988.

Frisk, G. V. (1994). Ocean and Seabed Acoustic: A Theory of Wave Propagation (Prentice-Hall, Englewood Cliffs, NJ), Chap. 5.

Gong, D., Glenn, S. M., Castelao, R., Kohut, J. T., and Schofield, O. (2008), "Characterizing summer time shelf-slope exchange processes on the New Jersey Shelf," in 2008 Ocean Sciences Meeting Abstracts, (ASLO/ AGU/TOS/ERF, Orlando, FL), pp. 137-137.

Headrick, R., Lynch, J. F. and the SWARM group (2000a). "Acoustic normal mode fluctuation statistics in the 1995 SWARM internal wave scattering experiment," J. Acoust. Soc. Am. 107, 201-220.
Headrick, R., Lynch, J. F. and the SWARM group (2000b), "Modeling mode arrivals in the 1995 SWARM experiment acoustic transmissions," J. Acoust. Soc. Am. 107, 221-236.

Lin, Y.-T., Newhall, A. E., Duda, T. F., Lermusiaux, P. F. J., and Haley, P. J. (2010). "Merging multiple-partial-depth data time series using objective empirical orthogonal function fitting," IEEE J. Ocean. Eng. 35, 710-721.

Lynch, J. F., Lin, Y.-T., Duda, T. F., and Newhall, A. E. (2010). "Acoustic ducting, reflection, refraction, and dispersion by curved nonlinear internal waves in shallow water," IEEE J. Oceanic Eng. 35, 12-27.

Markovsky, I., and Van Huffel, S. (2007). "Overview of total least-squares methods," Signal Processing. 87, 2283-2302.

Meyer, M., and Hermand, J.-P. (2006). "Backpropagation techniques in ocean acoustic inversion: Time reversal, retrogation and adjoint modelling-A review," in Acoustic Sensing Techniques for the Shallow Water Environment, edited by A. Caiti, N. R. Chapman, J.-P. Hermand, and S. M. Jesus (Springer, Netherlands), pp. 29-46.

Newhall, A. E., Duda, T. F., von der Heydt, K., Irish, J. D., Kemp, J. N., Lerner, S. A., Liberatore, S. P., Lin, Y.-T., Lynch, J. F., Maffei, A. R., Morozov, A. K., Shmelev, A., Sellers, C. J., and Witzell, W. E. (2007). "Acoustic and oceanographic observations and configuration information for the WHOI moorings from the SW06 experiment," Technical Report WHOI-2007-04 (Woods Hole Oceanographic Institution, Woods Hole, MA), Chap. 6.

Newhall, A. E., Lin, Y.-T., Lynch, J. F., Baumgartner, M. F., and Gawarkiewicz, G. (2012). "Long distance passive localization of vocalizing sei whales using an acoustic normal mode approach" J. Acoust. Soc. Am. 131, 1814-1825.

Park, C., Seong, W., Gerstoft, P., and Hodgkiss, W. S. (2010). "Geoacoustic inversion using backpropagation,” IEEE J. Ocean. Eng. 35, 722-731.

Rajan, S. D., and Becker, K. M. (2010). "Inversion for range-dependent sediment compressional-wave-speed profiles from modal dispersion data," IEEE J. Ocean. Eng. 35, 43-58.

Shang, E. C. (1985). "Source depth estimation in waveguides," J. Acoust. Soc. Am. 77, 1413-1418.

Shang, E. C., Clay, C. S., and Wang, Y. Y. (1985). "Passive harmonic source ranging in waveguides by using mode filter," J. Acoust. Soc. Am. 78, 172-175.

Shang, E. C. (1989). "An efficient high-resolution method of source localization processing in mode space," J. Acoust. Soc. Am. 86, 1960-1964.

Song, H., Kuperman, W. A., Hodgkiss, W. S., Gerstoft, P., and Kim, J. S. (2003). "Null broadening with snapshot-deficient covariance matrices in passive sonar," IEEE J. Ocean. Eng. 28, 250-261.

Tang, D. J., Moum, J. N., Lynch, J. F., Abbot, P., Chapman, R., Dahl, P. H., Duda, T. F., Gawarkiewicz, G., Glenn, S., Goff, J. A., Graber, H., Kemp, J., Maffei, A., Nash, J. D., and Newhall, A. (2007). "Shallow Water '06, A joint acoustic propagation/nonlinear internal wave physics experiment," Oceanography 20, 156-167.

Tappert, F. D., Nghiem-Phu, L., and Daubin, S. C. (1985). "Source localization using the PE method," J. Acoust. Soc. Am. 78, S30-S30.

Thode, A. M., D'Spain, G. L., and Kuperman, W. A. (2000). "MatchedField processing, source signature recovery, and geoacoustic inversion of blue whale vocalizations," J. Acoust. Soc. Am. 107, 1286-1300.

Tindle, C. T., Guthrie, K. M., Bold, G. E. J., Johns, M. D., Jones, D. Dixon, K. O., and Birdsall, T. G. (1978). "Measurements of the frequency dependence of normal modes," J. Acoust. Soc. Am. 64, 1178-1185.

Tolstoy, A. (1993). Matched Field Processing for Underwater Acoustics (World Scientific Publishing, Singapore).

Voltz, P., and Lu, I-T. (1994). "A time-domain backpropagating ray technique for source localization,” J. Acoust. Soc. Am. 95, 805-812.

Wunsch, C. (1996). The Ocean Circulation Inverse Problem (Cambridge University Press, Cambridge, UK), Chap. 3.

Yang, T. C. (1987). "A method of range and depth estimation by modal decomposition," J. Acoust. Soc. Am. 82, 1736-1745.

Yang, T. C. (1990). "Effectiveness of mode filtering: A comparison of matched-field and matched-mode processing," J. Acoust. Soc. Am. 87, 2072-2084. 